\title{
CPT-Based Probabilistic and Deterministic Assessment of In Situ Seismic Soil Liquefaction Potential
}

\author{
R. E. S. Moss, M.ASCE ; R. B. Seed, M.ASCE²; R. E. Kayen, M.ASCE ; J. P. Stewart, M.ASCE \\ A. Der Kiureghian, M.ASCE ${ }^{5}$; and K. O. Cetin, M.ASCE ${ }^{6}$
}

\begin{abstract}
This paper presents a complete methodology for both probabilistic and deterministic assessment of seismic soil liquefaction triggering potential based on the cone penetration test (CPT). A comprehensive worldwide set of CPT-based liquefaction field case histories were compiled and back analyzed, and the data then used to develop probabilistic triggering correlations. Issues investigated in this study include improved normalization of CPT resistance measurements for the influence of effective overburden stress, and adjustment to CPT tip resistance for the potential influence of "thin" liquefiable layers. The effects of soil type and soil character (i.e., "fines" adjustment) for the new correlations are based on a combination of CPT tip and sleeve resistance. To quantify probability for performancebased engineering applications, Bayesian "regression" methods were used, and the uncertainties of all variables comprising both the seismic demand and the liquefaction resistance were estimated and included in the analysis. The resulting correlations were developed using a Bayesian framework and are presented in both probabilistic and deterministic formats. The results are compared to previous probabilistic and deterministic correlations.
\end{abstract}

DOI: 10.1061/(ASCE)1090-0241(2006)132:8(1032)

CE Database subject headings: Seismic effects; Earthquakes; Cyclic loads; Liquefaction; In situ tests; Cone penetration tests; Probabilistic methods.

\section{Introduction}

Correlations based on in situ index tests are widely used in engineering practice to estimate the potential for "triggering" or initiation of seismically induced soil liquefaction. The cone penetration test (CPT) is a reliable in situ index test that has found widespread use as a tool for assessing the liquefaction resistance of potentially liquefiable soils. A number of CPT-based liquefaction triggering resistance curves have been suggested by previous researchers (e.g., Seed et al. 1983; Olsen 1984; Ishihara 1985; Jamiolkowski et al. 1985; Robertson and Campanella 1985; Franklin 1986; Seed and De Alba 1986; Shibata and Teparaska 1988; Sugawara 1989; Mitchell and Tseng 1990; Reyna 1991; Olsen and Koester 1995; Stark and Olson 1995; Rongxiang and Zhaoji

\footnotetext{
${ }^{1}$ Assistant Professor, Dept. of Civil and Environmental Engineering, California Polytechnic State Univ., San Luis Obispo, CA 93407 (corresponding author). E-mail: rmoss@calpoly.edu

${ }^{2}$ Professor, Dept. of Civil and Environmental Engineering, Univ. of California, Berkeley, CA.

${ }^{3}$ Researcher, United States Geological Survey, Menlo Park, CA.

${ }^{4}$ Associate Professor, Dept. of Civil and Environmental Engineering, Univ. of California, Los Angeles, CA.

${ }^{5}$ Professor, Dept. of Civil and Environmental Engineering, Univ. of California, Berkeley, CA.

${ }^{6}$ Professor, Middle East Technical University, Ankara, Turkey.

Note. Discussion open until January 1, 2007. Separate discussions must be submitted for individual papers. To extend the closing date by one month, a written request must be filed with the ASCE Managing Editor. The manuscript for this paper was submitted for review and possible publication on December 30, 2004; approved on December 9, 2005. This paper is part of the Journal of Geotechnical and Geoenvironmental Engineering, Vol. 132, No. 8, August 1, 2006. CASCE, ISSN 10900241/2006/8-1032-1051/\$25.00.
}

1995; Suzuki et al. 1995; Robertson and Wride 1998; Toprak et al. 1999; Juang et al. 2003; Idriss and Boulanger 2004).

The objective of this research is to provide as unbiased an assessment of CPT-based in situ soil liquefaction triggering potential as possible, and to assess the probability of liquefaction triggering for performance-based engineering applications. This is accomplished using a comprehensive worldwide CPT-based liquefaction field case history database compiled for this purpose (Moss et al. 2003b). One of the major challenges associated with the use of CPT data for liquefaction studies is the normalization of measured sleeve and tip resistance for the influence of effective overburden stress. We readdress this issue by applying cavity expansion methods to a prior empirically based normalization technique. Another challenge was related to processing the field case history data. We quantify the uncertainties associated with the various seismic demand and soil resistance variables. Moreover, we utilize a Bayesian framework to permit all types of uncertainties to be formally considered during the development of the model. The Bayesian framework uses structural reliability methods to estimate the probability of liquefaction conditional on penetration resistance and seismic demand. The results can be expressed as a mean and variance of the seismic demand needed to trigger liquefaction conditional on the penetration resistance, or, alternatively, the mean and variance of the penetration resistance needed for liquefaction conditional on the seismic demand.

\section{Threshold of Liquefaction Triggering}

The threshold of liquefaction triggering, or the liquefaction/ nonliquefaction boundary, has traditionally been located deterministically by engineering judgment. Based on the position and spread of the liquefaction and nonliquefaction data points, a curve 


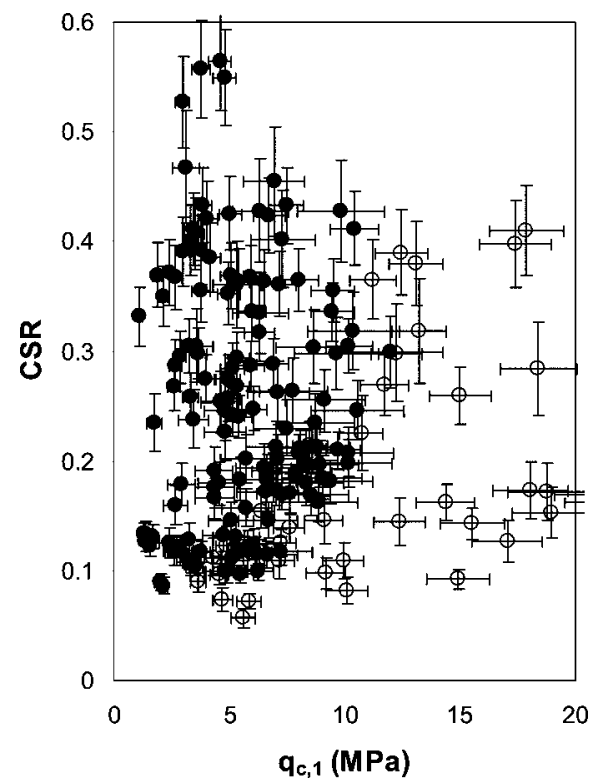

Fig. 1. Plot showing sample means and sample $( \pm 1) \mathrm{SD}$ of field case histories of liquefaction (closed circles) and nonliquefaction (open circles)

was drawn to estimate the threshold of liquefaction triggering. In this study, the threshold is located using engineering statistics, Bayesian updating, and reliability methods that are formulated for this particular problem (Cetin et al. 2002; Moss et al. 2003a).

Data collected and compiled for this study are shown in Fig. 1 as a plot of the sample mean of the liquefaction and nonliquefaction case histories with error bars showing \pm 1 sample standard deviation. The resistance is represented by the normalized CPT tip resistance $\left(q_{c, 1}\right)$ in megapascals $(\mathrm{MPa})$. The seismic demand is represented by the equivalent uniform cyclic stress ratio (CSR) as calculated using the simplified technique first proposed by Seed and Idriss (1971)

$$
\mathrm{CSR}=\frac{\tau_{\mathrm{avg}}}{\sigma_{v}^{\prime}}=0.65 \cdot \frac{a_{\mathrm{max}}}{g} \cdot \frac{\sigma_{v}}{\sigma_{v}^{\prime}} \cdot r_{d}
$$

In this study the equivalent uniform cyclic stress ratio was assumed to be the average or mean of an expansion of Eq. (1) which can be rewritten as Eq. (2). The variance of CSR can be calculated using Eq. (3), where the coefficient of variation $(\delta)$ is the normalized standard deviation, i.e., the standard deviation $(\sigma)$ divided by the mean $(\mu)$. Both Eqs. (2) and (3) are derived using a first-order Taylor series expansion of Eq. (1) about the mean point, including only the first two terms

$$
\begin{gathered}
\mu_{\mathrm{CSR}} \cong 0.65 \cdot \frac{\mu_{a_{\max }}}{g} \cdot \frac{\mu_{\sigma_{v}}}{\mu_{\sigma_{v}^{\prime}}} \cdot \mu_{r_{d}} \\
\delta_{\mathrm{CSR}}^{2} \cong \delta_{a_{\max }}^{2}+\delta_{r_{d}}^{2}+\delta_{\sigma_{v}}^{2}+\delta_{\sigma_{v}^{\prime}}^{2}-2 \cdot \rho_{\sigma_{v} \sigma_{v}^{\prime}} \cdot \delta_{\sigma_{v}} \cdot \delta_{\sigma_{v}^{\prime}}
\end{gathered}
$$

Eq. (3) indicates that the coefficient of variation of the CSR, the dependent variable, is a function of the coefficients of variation of the independent variables, which are the maximum horizontal ground acceleration $\left(a_{\max }\right)$, the total and effective vertical stresses $\left(\sigma_{v}\right.$ and $\left.\sigma_{v}^{\prime}\right)$, and the nonlinear shear mass participation factor $\left(r_{d}\right)$. The total and effective stresses are correlated variables, therefore in Eq. (3) the correlation coefficient term for these two variables is included. The other variables that comprise the CSR are assumed to have negligible correlation for the purpose of this study.

The coefficients of variation of the independent variables were estimated by Moss (2003). Full details are not presented here for brevity, but a brief synopsis follows:

1. The coefficients of variation of the total and effective stresses are calculated using Taylor-series expansions to account for the uncertainties in the water table depth and soil density.

2. The coefficient of variation of $a_{\max }$ is based on the quality of the locally available strong motion data.

3. The coefficient of variation of the moment magnitude is estimated based on a histogram of values reported in the literature.

4. The coefficient of variation of $r_{d}$ is estimated based on a statistical analysis of ground response by Cetin et al. (2004).

5. The coefficients of variation of the normalized tip resistance, $q_{c, 1}$, and the friction ratio, $R_{f}$, were calculated directly from the measured data within the "critical layer." We define the critical layer as being the most eminently liquefiable stratum in the profile, either because it was observed to have liquefied or is judged to be most susceptible to liquefaction based on current knowledge (further details are given in the following section).

As seen in Fig. 1 the variance of seismic demand tends to be greatest in the high CSR range and the variance of the resistance tends to greatest in the high $q_{c, 1}$ range. The region where liquefaction and nonliquefaction data points merge can be thought of as a "mixing" zone. In this zone lies the most likely threshold of liquefaction triggering.

\section{Critical Layer Selection}

As noted above, selection of the critical layer is necessary to the estimation of the means and standard deviations of $q_{c, 1}$ and $R_{f}$ for a given case history. Selecting the critical layer is an exercise in finding the soil stratum that is the "weakest link in the chain" from a liquefaction perspective. Finding this stratum requires considering the tip resistance and friction ratio jointly, with the addition of a boring $\log$ (if one is available) for soil classification. For most depositional environments this can be a simple matter of looking for the smallest continuous stretch of tip resistance with low friction ratio that agrees with the boring log in terms of the material texture (i.e., cohesive versus cohesionless soils). This can be a difficult task for fluvial deposits, which tend to be thin, interbedded, and discontinuous both horizontally and vertically. A second method for identifying a critical layer is to calculate the profile of cyclic resistance ratio (CRR) based on existing correlations and take the critical layer as the domain with the smallest CRR. This method is used for the case histories where multiple candidates for the critical layer exist and it is unclear which of these layers liquefied or did not liquefy.

We define a liquefaction/nonliquefaction field case history differently than previous investigators. We define a single case history as a critical layer that experienced strong ground shaking during an earthquake. All CPT soundings that registered that critical layer were used in assessing the mean and variance of $q_{c, 1}$ and $R_{f}$. In contrast, previous studies often took a case history as the critical layer from an individual CPT sounding, hence multiple case histories could be present for a given site if multiple soundings were available. We believe our procedure provides an inventory of case histories that are relatively statistically independent of each other, which is desirable for an unbiased database. 
One issue that is not commonly addressed in liquefaction correlations is that the in situ data are usually acquired following strong ground shaking. Particularly for the liquefied cases, the soil has likely densified due to postliquefaction reconsolidation. Previous studies have investigated pre- and postearthquake soil properties in deposits subject to liquefaction. In several of those studies, soil properties were observed to not change noticeably (e.g., Inagaki et al. 1996; Boulanger et al. 1997); whereas in others small increases in penetration resistance were observed (e.g., Chameau et al. 1991; Frost et al. 1993; Shibata et al. 1996). Sites with noticeable changes in properties consisted of recent artificial fills typically having relatively low penetration resistance standard penetration test (SPT) $(N<10)$, whereas sites without soil property changes consisted of fills and alluvia having somewhat higher penetration resistance (SPT $N>10$ ).

These observations are consistent with critical state principles, which would predict that volume change from postliquefaction reconsolidation would increase with the state parameter (i.e., increase with decreasing relative density for a fixed confinement). CPT measurements are in turn sensitive to the state parameter because tip resistance is positively correlated with relative density for "clean" sands (Schmertmann 1978).

Given the available data on pre- and postearthquake penetration resistance of liquefied sediments, we anticipate that the triggering limit-state, or threshold, will not be significantly affected by postliquefaction reconsolidation because:

1. Near the limit-state the relative state parameter index of the soils is in the range of 0 to -0.65 (Idriss and Boulanger 2004) indicating that they would not be expected to have significantly densified.

2. Nonliquefied soils will have lower values of the state parameter and hence would be expected to have been even less affected by densification.

3. Highly contractive soils that would be expected to produce the greatest changes in penetration resistance have little impact on the position of the limit-state function in a Bayesiantype analysis.

\section{Data Screening, Adjustment, and Correction}

A thorough and proper treatment of the data requires that it be screened, adjusted, and corrected for various effects. The purpose of the screen is to separate liquefaction of cohesionless soils from cyclic softening of clays, both of which can produce observable ground failure in the field. Screening has traditionally been performed using the "Chinese criteria" (Wang 1979; Seed and Idriss 1982), although recent research suggests some modification to those criteria may be warranted (Andrews and Martin 2000; Seed et al. 2003; Boulanger and Idriss 2004; Bray et al. 2004).

Corrections and adjustments to the penetration resistance measurements are needed for the influence of effective overburden stress, and for the influence of the "thinness" of a liquefiable layer. A correction to CSR is needed for the effect of the duration of strong ground shaking. Moreover, the calculation of CSR must account for the nonlinear shear mass participation factor $\left(r_{d}\right)$. Each of these issues is described in sections that follow.

\section{Screening}

Cohesive soils are not susceptible to liquefaction, but may experience shear failure that is exacerbated by cyclic softening. These soils can produce observable ground failure that appears similar

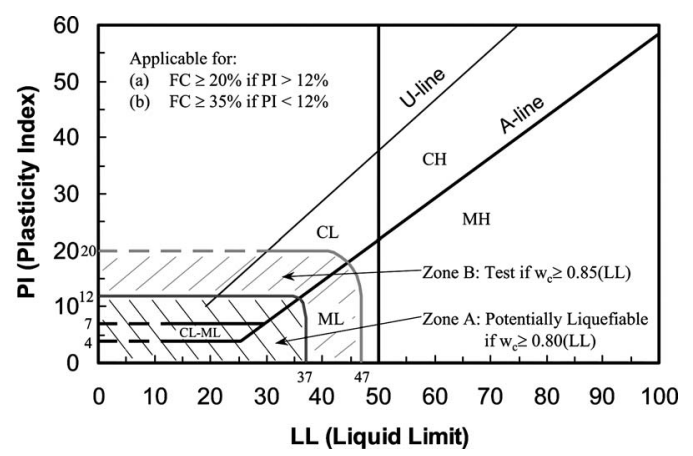

Fig. 2. Modified "Atterberg limits" chart showing recommendations regarding assessment of soil types considered liquefiable [Seed et al. (2003) with permission]

to ground failure from liquefaction, such as building tilting, punching, and settlement. However, there are fundamental differences between the cyclic behavior of cohesive and cohesionless soils, including lower peak pore pressure ratios and higher postsoftening shear strengths in cohesive materials (Boulanger and Idriss 2004). Ground failure in cohesive materials is typically only observed when there is a large asymmetrical driving shear stress $\left(K_{\alpha}\right)$, and is best analyzed by comparing the undrained cyclic shear strength with applied shear stresses (cyclic+static). Several such cases were observed following the 2001 Kocaeli, Turkey, and 2001 Chi-Chi, Taiwan, earthquakes (e.g., Bray et al. 2004; Chu et al. 2004), and were evaluated during the compilation of the case history database. Since the limit-state function in this study is intended for application to predominantly cohesionless soils, it was not appropriate to include these cases in the analysis.

These screened cases exhibited a combination of low tip resistance $\left(q_{c, 1}\right.$ less than $\left.\sim 5 \mathrm{MPa}\right)$ and high friction ratio $\left(R_{f}\right.$ greater than $\sim 3 \%$ ), which are typical of cohesive soils. These cases, most noticeably the nonliquefaction cases, plotted far left of previous triggering curves, suggesting that the soils may have been too clay rich to liquefy. In addition to the CPT measurements, it was necessary to use soil texture information from SPT samples for screening. The criteria for screening these cases were based on research of fines content and plasticity in relation to liquefaction susceptibility (Yamamuro and Lade 1998; Guo and Prakash 1999; Youd and Gilstrap 1999; Andrews and Martin 2000; Perlea 2000; Andrianopoulos et al. 2001; Polito 2001; Boulanger and Idriss 2004; Bray et al. 2004). Zone A, shown graphically in Fig. 2, is the envelope of potentially liquefiable materials used in this study for sites where laboratory data were available. The case histories that plot within Zone A, and are therefore included in the database are: Wu Feng Site A from the Chi-Chi event [liquid limit $(L L)=30$, plasticity index $(P I)=5]$, the Tientsin sites from the Tangshan event $(\mathrm{LL} \sim 30, \mathrm{PI} \sim 10)$, Balboa Site $\mathrm{C} \quad(\mathrm{LL}=30$, $\mathrm{PI}=11)$, Malden Site D $(\mathrm{LL}=31, \mathrm{PI}=12)$ from the Northridge event, Whiskey Springs Site $1(\mathrm{LL}=22.5, \mathrm{PI}=2)$ from the Borah Peak event, Adapazari Site J $(\mathrm{LL}=33, \mathrm{PI}=6)$, and Site K $(\mathrm{LL}=35, \mathrm{PI}=9)$ from the Kocaeli event.

Another potentially significant issue relates to cases where liquefaction may have occurred at depth but was not manifest at the ground surface due to relatively thick and continuous overlying nonliquefiable strata. The issue of liquefaction manifestation at the ground surface is discussed by Ishihara (1985) and Youd and Garris (1995). The results from these studies were used to screen sites that were reported as nonliquefied but found to be liquefiable 


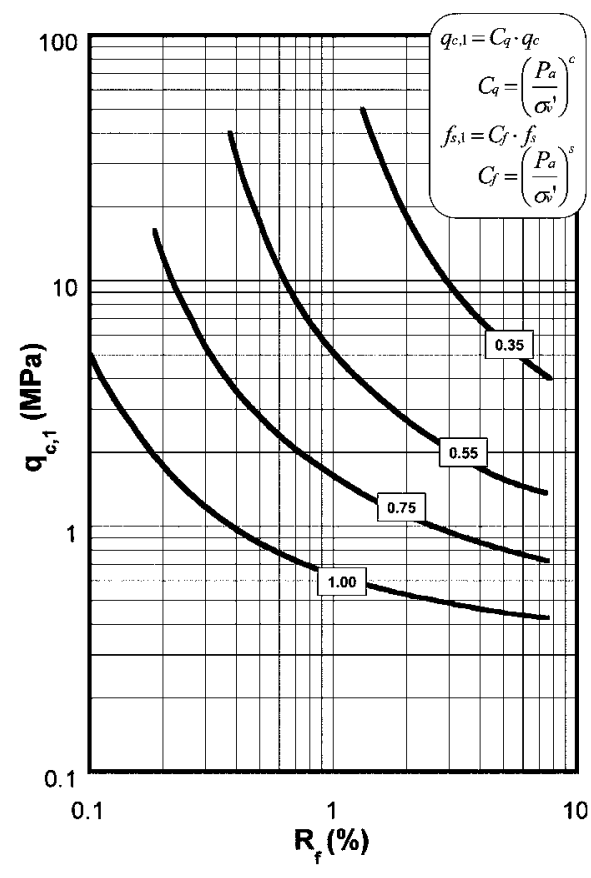

Fig. 3. Proposed CPT normalization exponent curves from Moss et al. (2006). These curves are labeled with their respective normalization exponent, $c$, values. Iterative normalization exponent procedure, Eq. (5), can be used instead of this figure to calculate normalization exponent. It is recommended that tip and sleeve resistance be normalized equivalently $(s=c)$.

in terms of the index measurements, have overlying nonliquefiable material that fits the Ishihara thickness criteria, showed no surface manifestation of liquefaction, have level ground conditions, and were not subject to ground oscillations. For example, at a level site with a $2 \mathrm{~m}$ thick liquefiable layer experiencing ground shaking with peak ground acceleration $(\mathrm{PGA})<0.2 \mathrm{~g}$, an overlying nonliquefiable layer of approximately $2 \mathrm{~m}$ could eliminate all surface manifestation of liquefaction.

This study focused on free-field sites, or sites with no significant static shear stresses. The database includes sites with relatively level ground conditions, but excludes sites where there is sloping ground, or a structure (e.g., buildings, embankments, etc.) that can induce static shear stress.

\section{Normalization}

Effective overburden stress can profoundly influence CPT measurements (Olsen and Mitchell 1995). This effect is typically accounted for by normalizing the tip resistance measured at a given depth and vertical effective stress to a reference effective stress of 1 atm. A complete discussion of CPT normalization for effective overburden stress can be found in Moss et al. (2006). In that paper cavity expansion models (Ladanyi and Johnston 1974; Yu 2000; Cao et al. 2001; Salgado and Randolf 2001; Boulanger 2003) were used in conjunction with field and laboratory test data (Olsen and Mitchell 1995) to estimate appropriate levels of normalization as a function of the measured tip resistance and friction ratio. The discussion in this paper is limited, for brevity, to the application of the proposed normalization technique.

Fig. 3 shows normalization exponent curves as a function of friction ratio and tip resistance. These curves define the exponent used to normalize the tip resistance for a given level of overburden stress, using the following equation:

$$
q_{c, 1}=C_{q} \cdot q_{c}
$$

where

$$
C_{q}=\left(\frac{P_{a}}{\sigma_{v}^{\prime}}\right)^{c}
$$

where $q_{c, l}=$ normalized tip resistance $(\mathrm{MPa}) ; C_{q}=$ tip normalization factor; $q_{c}=$ raw tip resistance $(\mathrm{MPa}) ; P_{a}=$ reference stress $\left(1 \mathrm{~atm}=101.325 \mathrm{kPa}=1.033 \mathrm{~kg} / \mathrm{cm}^{2}=14.696 \mathrm{psi}=1.058 \mathrm{ft}^{2}\right) \quad$ in compatible units; $\sigma_{v}^{\prime}=$ effective overburden stress (same units as $P_{a}$ ); and $c=$ normalization exponent. In Eq. (4), $\mathrm{C}_{q}$ should not exceed a value of 1.7 .

In previous practice the normalization exponent, $c$, has generally been taken as 0.5 (Liao and Whitman 1986). This value of 0.5 is the average exponent value of the response of different soil types to overburden, and therefore neglects the inherent variability. It was found that including this variability, by using the proposed normalization procedure, resulted in a much better statistical fit (as measured by the model error term) of the liquefaction triggering curves. A full discussion of this normalization scheme, its empirical and theoretical basis, and how this applies to other in situ tests such as the SPT can be found in Moss et al. (2006).

For calculation purposes the normalization exponent curves in Fig. 3 can be calculated using the iterative equation

$$
c=f_{1} \cdot\left(\frac{R_{f}}{f_{3}}\right)^{f_{2}}
$$

where $f_{1}=x_{1} \cdot q_{c}^{x 2} ; \quad f_{2}=-\left(y_{1} \cdot q_{c}^{y 2}+y_{3}\right) ; \quad f_{3}=\operatorname{abs}\left[\log \left(10+q_{c}\right)\right]^{z 1}$; $x_{1}=0.78 ; x_{2}=-0.33 ; y_{1}=-0.32 ; y_{2}=-0.35 ; y_{3}=0.49 ; z_{1}=1.21$; $c=$ tip normalization exponent; $q_{c}=$ raw tip resistance $(\mathrm{MPa})$; $R_{f}=$ friction ratio (the ratio of sleeve to tip resistance, $f_{s} / q_{c}$, in percent); and $f_{1}, f_{2}, f_{3}, x_{1}, x_{2}, y_{1}, y, y_{3}$, and $z_{1}=$ parameters of the equation. To normalize the tip resistance appropriately, an iterative procedure is necessary. The iterative procedure involves the following steps:

1. An initial estimate of the normalization exponent is found using raw tip measurements, friction ratio, and Fig. 3 or Eq. (5);

2. The tip is then normalized using Eq. (4) (note: friction ratio will not change when tip and sleeve are normalized equivalently);

3. A revised estimate of the normalization exponent is found using the normalized tip resistance and Fig. 3 or Eq. (5), which is compared to the initial normalization exponent estimate; and

4. The procedure is repeated until an acceptable convergence tolerance is achieved.

For most soils this process usually requires only two iterations to converge. (In Excel, the Solver Add-In in the Analysis Toolpack can be useful for this iterative procedure in spreadsheet calculations.) It is recommended that the tip and sleeve be normalized equivalently.

\section{Magnitude Correlated Duration Weighting Factor $\left(\mathrm{DWF}_{M}\right)$}

The results presented in this study include the correction of equivalent uniform CSR for duration (or number of equivalent 


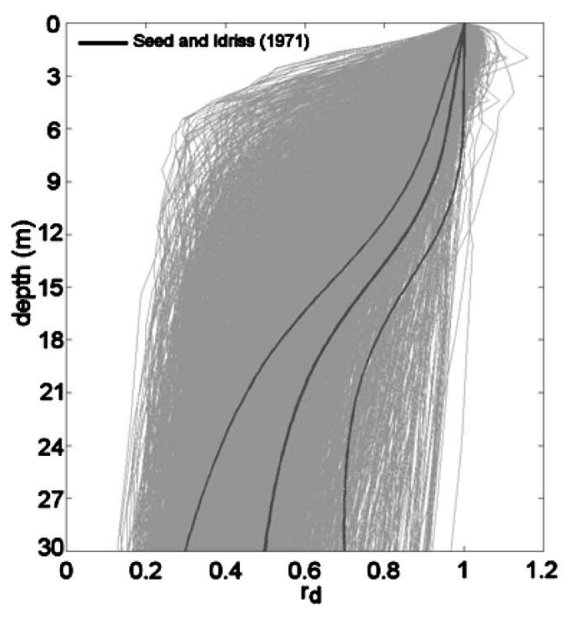

a)

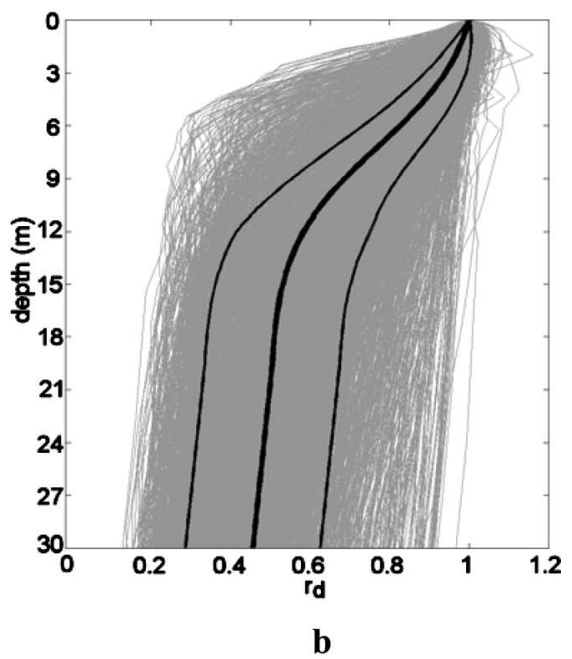

Fig. 4. $r_{d}$ results from response analyses for 2,153 combinations of site conditions and ground motions, superimposed with heavier lines showing; (a) earlier recommendations of Seed and Idriss (1971); (b) mean and +1 SD values for 2,153 cases analyzed (Cetin et al. 2004, ASCE) [Note: CSR was calculated in this current study using exclusively $r_{d}$ as proposed by Cetin et al. (2004), and older $r_{d}$ recommendations are not compatible with this study. The Cetin et al. (2004) method allows for forward analysis using either direct ground response analyses, or "simplified" $r_{d}$-based assessment of in situ CSR.]

cycles) to $\mathrm{CSR}^{*}$, representing the CSR for a duration typical of an "average" event of $M_{W}=7.5$. This is accomplished using a magnitude-correlated duration weighting factor $\left(\mathrm{DWF}_{M}\right)$

$$
\mathrm{CSR}^{*}=\mathrm{CSR}_{M_{w}=7.5}=\frac{\mathrm{CSR}_{M_{w}=?}}{\mathrm{DWF}_{M_{w}}}
$$

The duration weighting factor is somewhat controversial, and has previously been developed using different approaches (e.g., cyclic laboratory testing and/or field case history data) by a number of investigators. Cetin et al. (2004) regressed the $\mathrm{DWF}_{M}$ from a SPT-based liquefaction database that included events covering a wide range of moment magnitudes. These results were found to be in good agreement with previously published lower-bound results by Youd et al. (2001), and also consistent with laboratory results published by Idriss (1999) and Liu et al. (2001). The present CPT-based study was lacking a wide enough magnitude range to accurately discern the $\mathrm{DWF}_{M}$ in a similar manner, therefore the Cetin et al. (2004) $\mathrm{DWF}_{M}$ results were used. The recommended $\mathrm{DWF}_{M}$ can be represented by the equation

$$
\mathrm{DWF}_{M}=17.84 \cdot M_{w}^{-1.43}
$$

Eq. (7) is valid for $M_{w}=5.5-8.5$.

\section{Nonlinear Shear Mass Participation Factor $\left(r_{d}\right)$}

The nonlinear shear mass participation factor $\left(r_{d}\right)$ accounts for nonlinear ground response in the soil column overlying the depth of interest. This factor, previously proposed by various researchers, was reassessed by Cetin et al. (2004) using ground response analyses. In this work 2,153 ground response analyses were run using 50 sites and 42 ground motions. The results (Fig. 4) were regressed to evaluate the median $r_{d}$ for a given depth, peak ground acceleration, and moment magnitude. The variance was estimated from the dispersion of the simulations. The median $r_{d}$ results can calculated using the following equations:

for $d<20 \mathrm{~m}$

$$
\begin{aligned}
r_{d}\left(d, M_{w}, a_{\max }\right) \\
=\frac{\left[1+\frac{-9.147-4.173 \cdot a_{\max }+0.652 \cdot M_{w}}{10.567+0.089 \cdot e^{0.089 \cdot\left(-d \cdot 3.28-7.760 \cdot a_{\max }+78.576\right)}}\right]}{\left[1+\frac{-9.147-4.173 \cdot a_{\max }+0.652 \cdot M_{w}}{10.567+0.089 \cdot e^{0.089 \cdot\left(-7.760 \cdot a_{\max }+78.576\right)}}\right]}
\end{aligned}
$$

and for $d \geqslant 20 \mathrm{~m}$

$$
\begin{aligned}
r_{d}\left(d, M_{w}, a_{\max }\right) & \\
= & \frac{\left[1+\frac{-9.147-4.173 \cdot a_{\max }+0.652 \cdot M_{w}}{10.567+0.089 \cdot e^{0.089 \cdot\left(-d \cdot 3.28-7.760 \cdot a_{\max }+78.567\right)}}\right]}{\left[1+\frac{-9.147-4.173 \cdot a_{\max }+0.652 \cdot M_{w}}{10.567+0.089 \cdot e^{0.089 \cdot\left(-7.760 \cdot a_{\max }+78.567\right)}}\right]} \\
& -0.0014 \cdot(d \cdot 3.28-65)
\end{aligned}
$$

where $d=$ depth in meters at the midpoint of the critical layer; $M_{w}=$ moment magnitude; and $a_{\max }=$ peak ground acceleration in units of gravity. The standard deviation for $r_{d}$ is as follows:

for $d<12.2 \mathrm{~m}$

$$
\sigma_{r_{d}}(d)=(d \cdot 3.28)^{0.864} \cdot 0.00814
$$

and for $d \geqslant 12.2 \mathrm{~m}$

$$
\sigma_{r_{d}}(d)=40^{0.864} \cdot 0.00814
$$

Eqs. (8)-(11), from Cetin (2000) are slightly different than the equations presented in Cetin et al. (2004), in that they lack the shear wave velocity $\left(V_{s}\right)$ term. The majority of the case histories in the CPT database did not have shear wave velocity measurements, therefore instead of introducing an inferred or indirectly estimated shear wave velocity (and its associated uncertainty) into the analysis, the $r_{d}$ correlation without $V_{s}$ was used. If shear wave velocity measurements are available, then the equations including the shear wave velocity term (Cetin et al. 2004) can be used to calculate $r_{d}$. 


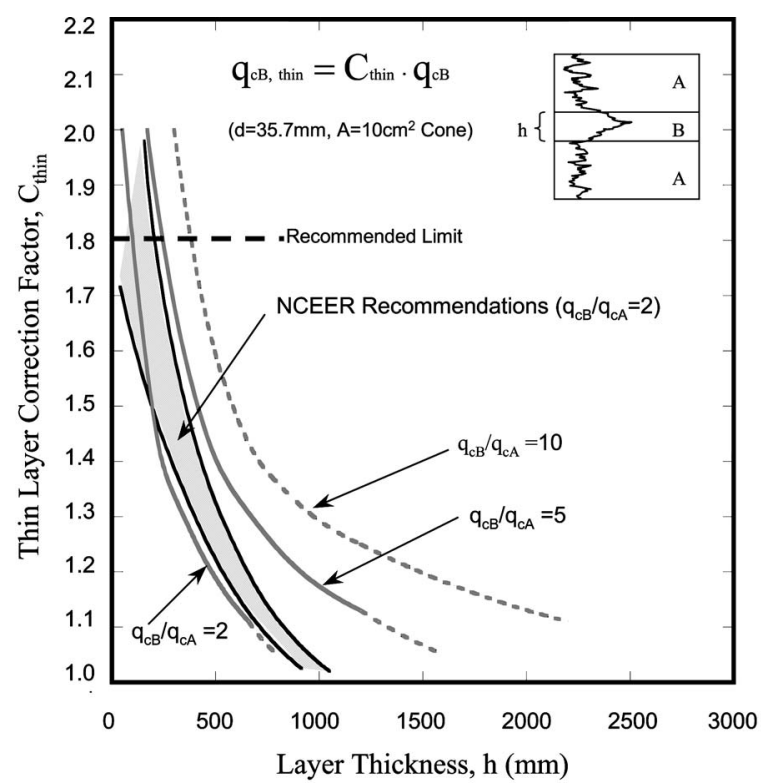

Fig. 5. Recommended thin layer corrections, shown along with previous NCEER working group recommendations (shaded zone). Upper limit of $C_{\text {thin }} \leqslant 1.8$ is suggested for forward analyses.

This method of estimating $r_{d}$ was used exclusively in this analysis, since previous methods for calculating $r_{d}$ are not compatible with the proposed CPT correlation because of potential biases. Because the proposed CPT correlation was based on this new $r_{d}$ method that includes ground response analysis, shear stresses computed at depth from site-specific ground response analyses can be used in applications.

\section{Thin Layer Correction}

As discussed in Youd et al. (2001), previous studies have shown that tip resistance can be influenced by the presence of softer soils above and below a liquefiable layer. Youd et al. (2001) showed a comparison of curves from Robertson and Fear (1995) with field data presented at the NCEER workshop by G. Castro and P. Robertson. The conclusion of the workshop was that the curves appear to represent the appropriate trend but suggest too high of a correction. The field data was used to present a lower bound for the recommended range of corrections.

We further investigate the thin layer correction using the simplified elastic solution by Vreugdenhil et al. (1994), which was also used by Robertson and Fear (1995) to establish their thin layer correction curves. The elastic solution was originally developed to evaluate two conditions: (1) stiff thin layers embedded in softer surrounding material; and (2) soft thin layers embedded in stiffer surrounding material. The first case of the stiff thin layer is the case that is germane to the problem of liquefaction. An example is fluvial environments where it is common to find interbedded deposits of granular and fine materials. The granular deposits would give a much higher tip resistance than the surrounding fine deposits, thereby producing a stiff, thin, potentially liquefiable layer within softer surrounding material.

We calibrated the elastic solution of Vreugdenhil et al. (1994) using chamber data from Kurup et al. (1994). Correction curves were then calculated for different ratios of tip resistance and compared to field data from 22 field sites. Shown in Fig. 5 is the recommended thin layer correction compared to the range recom- mended in Youd et al. (2001). Corrections are shown for $q_{c B} / q_{c A}$ ratios of 2 and 5 with an upper limit of 10 . These curves compare favorably with the NCEER workshop recommendations for $q_{c B} / q_{c A}=2$. The results also follow the same trends and magnitudes for loose sands as presented by Meyerhoff (1976) for piles driven through a profile of softer over stiffer soils. These thin layer corrections are for a standard cone with a diameter of $35.7 \mathrm{~mm}$ and a tip area of $10 \mathrm{~cm}^{2}$.

Thin layer correction only applied to four out of the 188 field case histories in the liquefaction/nonliquefaction case history database, but is presented here for practitioners who may have need for the corrections when dealing with similar field conditions. The thin layer correction was conservatively capped at 1.5 for the case histories in this study, and an upper limit of 1.8 is recommended for practice.

\section{Data Quality Assessment}

After the case histories were subjected to the screening, adjustments, and corrections discussed above, they were classified according to the quality of the available data. Four classes of data, A-D, were used to group the data, with $\mathrm{D}$ being substandard and therefore excluded from the final case history database. The criteria for the data classes are as follows:

1. Class A

a. Original CPT trace with $q_{c}$ and $f_{s} / R_{f}$, using an ASTM D3441 and D5778 spec. cone.

b. $\delta_{\mathrm{CSR}} \leqslant 0.20$ [i.e., square root of Eq. (3)].

2. Class B

a. Original CPT trace with $q_{c}$ and $f_{s} / R_{f}$, using an ASTM D3441 and D5778 spec. cone.

b. $\quad 0.20<\delta_{\mathrm{CSR}} \leqslant 0.35$.

3. Class $\mathrm{C}$

a. Original CPT trace with $q_{c}$ and $f_{s} / R_{f}$, but using a nonstandard cone (e.g., Chinese cone or mechanical cone).

b. No sleeve data but fines content $(\mathrm{FC}) \leqslant 5 \%$ (i.e., "clean" sand).

4. Class D

c. $\quad 0.35<\delta_{\mathrm{CSR}} \leqslant 0.50$.

a. Not satisfying the criteria for Classes A, B, or C.

From the over 500 possible case histories, 188 achieved a Class $\mathrm{C}$ or better and were included in the field case history database that documents 18 different earthquakes spanning 4 decades. The earthquakes included are; 1964 Niigata, 1968 Inangahua, 1975 Haicheng, 1976 Tangshan, 1977 Vrancea, 1979 Imperial Valley, 1980 Mexicali, 1981 Westmorland, 1983 NihonkaiChubu, 1983 Borah Peak, 1987 Elmore Ranch, 1987 Superstition Hills, 1987 Edgecumbre, 1989 Loma Prieta, 1994 Northridge, 1995 Hyogoken-Nambu (Kobe), 1999 Kocaeli, and 1999 Chi-Chi earthquakes. The database was compiled into a single report (Moss et al. 2003b), in which each case history includes at least two worksheets documenting the measured and calculated values used in the analysis, the CPT trace(s) evaluated, the references cited, and other pertinent information. Table 1 shows a summary of means and standard deviations used in the analysis.

\section{Bayesian Framework}

A Bayesian framework utilizing structural reliability methods was used to evaluate the processed data and develop a correlation between the seismic demand and resistance variables with respect to observed liquefaction/nonliquefaction. 


\begin{tabular}{|c|c|c|c|c|c|c|c|c|c|c|c|c|c|c|c|}
\hline Earthquake & Site & $M_{w}$ & Liquefied? & $\begin{array}{l}\text { Data } \\
\text { class }\end{array}$ & $\begin{array}{l}\text { Crit. } \\
\text { depth } \\
\text { range } \\
(\mathrm{m})\end{array}$ & $\begin{array}{l}\text { Depth } \\
\text { to } \\
\text { GWT } \\
\text { (m) }\end{array}$ & $\begin{array}{c}\sigma_{\mathrm{vo}} \\
(\mathrm{kPa})\end{array}$ & $\begin{array}{c}\sigma_{\mathrm{vo}}^{\prime} \\
(\mathrm{kPa})\end{array}$ & $\begin{array}{c}a_{\max } \\
(\mathrm{g})\end{array}$ & $r_{d}$ & CSR & $c$ & $\begin{array}{c}q_{c 1} \\
(\mathrm{MPa})\end{array}$ & $\begin{array}{l}R_{f} \\
(\%)\end{array}$ & Reference \\
\hline \multirow[t]{3}{*}{1964 Niigata, Japan } & $\mathrm{D}$ & $7.50 \pm 0.11$ & Yes & B & $2.7-6.0$ & 1.12 & $47.94 \pm 10.56$ & $32.44 \pm 4.16$ & $0.16 \pm 0.03$ & $0.95 \pm 0.05$ & $0.15 \pm 0.05$ & 0.45 & $6.24 \pm 1.73$ & $1.14 \pm 0.65$ & \multirow{3}{*}{$\begin{array}{l}\text { Farrar (1990); } \\
\text { Ishihara and Koga (1981) }\end{array}$} \\
\hline & $\mathrm{E}$ & & Yes & B & $1.8-4.8$ & 0.67 & $68.00 \pm 12.82$ & $44.46 \pm 4.94$ & $0.16 \pm 0.03$ & $0.92 \pm 0.07$ & $0.15 \pm 0.04$ & 0.47 & $4.56 \pm 1.13$ & $1.22 \pm 0.60$ & \\
\hline & $\mathrm{F}$ & & No & B & $1.7-2.2$ & 1.70 & $31.95 \pm 2.13$ & $29.50 \pm 2.38$ & $0.16 \pm 0.03$ & $0.97 \pm 0.04$ & $0.11 \pm 0.02$ & 0.38 & $9.39 \pm 8.97$ & $1.40 \pm 1.81$ & \\
\hline \multirow[t]{2}{*}{$\begin{array}{l}1968 \text { Inangahua, } \\
\text { New Zealand }\end{array}$} & Three Channel Flat & $7.40 \pm 0.11$ & Yes & $\mathrm{C}$ & $0.5-2.5$ & 0.10 & $29.00 \pm 6.60$ & $15.27 \pm 3.37$ & $0.40 \pm 0.10$ & $0.97 \pm 0.03$ & $0.48 \pm 0.19$ & 0.53 & $2.84 \pm 0.96$ & $1.39 \pm 0.70$ & \multirow{2}{*}{$\begin{array}{l}\text { Ooi (1987); Dowrick } \\
\text { and Sritharan (1968); Zhao } \\
\text { et al. (1997) }\end{array}$} \\
\hline & Reedy’s Farm & & Yes & B & $1.0-1.8$ & 0.10 & $26.66 \pm 2.68$ & $14.10 \pm 2.51$ & $0.20 \pm 0.05$ & $0.98 \pm 0.03$ & $0.24 \pm 0.08$ & 0.65 & $2.62 \pm 0.69$ & $0.79 \pm 0.52$ & \\
\hline \multirow[t]{5}{*}{1975 Haicheng, China } & Chemical Fiber Site & $7.30 \pm 0.11$ & Yes & $\mathrm{C}$ & $7.8-12.0$ & 1.52 & $179.35 \pm 14.57$ & $97.14 \pm 7.28$ & $0.15 \pm 0.05$ & $0.71 \pm 0.16$ & $0.13 \pm 0.06$ & 0.85 & $1.37 \pm 0.64$ & $0.76 \pm 0.43$ & \multirow{5}{*}{$\begin{array}{l}\text { EarthTech (1985); Arulanandan } \\
\text { et al. (1986); Shengcong } \\
\text { and Tatsuaoka (1984) }\end{array}$} \\
\hline & Const. Com. Building & & Yes & $\mathrm{C}$ & $5.5-7.5$ & 1.52 & $116.45 \pm 6.81$ & $67.60 \pm 4.94$ & $0.15 \pm 0.05$ & $0.83 \pm 0.11$ & $0.14 \pm 0.05$ & 0.92 & $0.77 \pm 0.14$ & $1.37 \pm 0.27$ & \\
\hline & Guest House & & Yes & $\mathrm{C}$ & $8.0-9.5$ & 1.52 & $158.08 \pm 6.05$ & $87.15 \pm 5.42$ & $0.15 \pm 0.05$ & $0.75 \pm 0.15$ & $0.13 \pm 0.05$ & 0.86 & $0.97 \pm 0.18$ & $1.08 \pm 0.41$ & \\
\hline & 17th Middle School & & Yes & $\mathrm{C}$ & $4.5-11.0$ & 1.52 & $136.46 \pm 19.79$ & $75.34 \pm 8.40$ & $0.15 \pm 0.05$ & $0.79 \pm 0.13$ & $0.14 \pm 0.06$ & 0.87 & $0.92 \pm 0.29$ & $1.02 \pm 0.44$ & \\
\hline & Paper Mill & & Yes & $\mathrm{C}$ & $3.0-5.0$ & 1.52 & $70.20 \pm 6.46$ & $45.87 \pm 4.44$ & $0.15 \pm 0.05$ & $0.91 \pm 0.08$ & $0.14 \pm 0.05$ & 0.77 & $1.16 \pm 0.31$ & $1.28 \pm 0.56$ & \\
\hline \multirow{15}{*}{$\begin{array}{l}1976 \text { Tangshan, } \\
\text { China }\end{array}$} & Tientsin Y21 [1] & $8.00 \pm 0.09$ & Yes & $\mathrm{C}$ & $4.5-5.25$ & 1.00 & $89.63 \pm 3.45$ & $51.61 \pm 4.02$ & $0.08 \pm 0.03$ & $0.91 \pm 0.09$ & $0.09 \pm 0.04$ & 0.76 & $0.97 \pm 0.42$ & $2.50 \pm 1.84$ & \multirow{15}{*}{$\begin{array}{l}\text { [1] Arulanandan et al. (1982); } \\
\text { [2] Zhou and Zhang (1979); } \\
\text { Shibata and Teparaska (1988) }\end{array}$} \\
\hline & Tientsin Y24 [1] & & Yes & $\mathrm{C}$ & $3.5-4.5$ & 0.20 & $75.40 \pm 4.09$ & $38.12 \pm 3.34$ & $0.09 \pm 0.04$ & $0.93 \pm 0.08$ & $0.11 \pm 0.05$ & 0.70 & $3.64 \pm 0.632$ & $0.72 \pm 0.15$ & \\
\hline & Tientsin Y28 [1] & & Yes & $\mathrm{C}$ & $1.0-3.0$ & 0.20 & $37.40 \pm 6.50$ & $19.74 \pm 3.13$ & $0.09 \pm 0.04$ & $0.97 \pm 0.04$ & $0.11 \pm 0.05$ & 0.68 & $2.78 \pm 0.87$ & $0.78 \pm 0.33$ & \\
\hline & Tientsin Y29 [1] & & Yes & $\mathrm{C}$ & $2.8-3.8$ & 1.00 & $59.70 \pm 3.66$ & $37.14 \pm 2.80$ & $0.08 \pm 0.03$ & $0.95 \pm 0.06$ & $0.09 \pm 0.04$ & 0.74 & $1.93 \pm 0.22$ & $0.91 \pm 0.59$ & \\
\hline & T1 Tangshan District [2] & & Yes & $\mathrm{C}$ & $4.1-5.8$ & 3.70 & $82.95 \pm 8.95$ & $70.69 \pm 4.26$ & $0.40 \pm 0.16$ & $0.86 \pm 0.09$ & $0.26 \pm 0.11$ & 0.75 & $5.95 \pm 1.29$ & $0.38 \pm 0.38$ & \\
\hline & T2 Tangshan District [2] & & Yes & $\mathrm{C}$ & $2.3-4.3$ & 1.30 & $58.80 \pm 4.77$ & $39.18 \pm 2.93$ & $0.40 \pm 0.16$ & $0.92 \pm 0.06$ & $0.36 \pm 0.15$ & 0.78 & $3.79 \pm 1.56$ & $0.38 \pm 0.38$ & \\
\hline & T8 Tangshan District [2] & & Yes & $\mathrm{C}$ & $4.5-6.0$ & 2.00 & $93.75 \pm 5.42$ & $61.87 \pm 3.54$ & $0.40 \pm 0.16$ & $0.84 \pm 0.10$ & $0.33 \pm 0.14$ & 0.72 & $8.03 \pm 3.68$ & $0.38 \pm 0.38$ & \\
\hline & T10 Tangshan District [2] & & Yes & $\mathrm{C}$ & $6.5-0.8$ & 1.45 & $150.50 \pm 11.37$ & $84.77 \pm 5.92$ & $0.40 \pm 0.16$ & $0.73 \pm 0.14$ & $0.34 \pm 0.15$ & 0.75 & $5.90 \pm 1.01$ & $0.38 \pm 0.38$ & \\
\hline & T19 Tangshan District [2] & & Yes & $\mathrm{C}$ & $2.0-4.5$ & 1.10 & $59.26 \pm 8.22$ & $38.17 \pm 3.71$ & $0.20 \pm 0.08$ & $0.94 \pm 0.06$ & $0.19 \pm 0.08$ & 0.69 & $8.00 \pm 1.74$ & $0.38 \pm 0.38$ & \\
\hline & T22 Tangshan District [2] & & Yes & $\mathrm{C}$ & $7.0-8.0$ & 0.80 & $141.98 \pm 5.45$ & $76.25 \pm 4.90$ & $0.20 \pm 0.08$ & $0.80 \pm 0.13$ & $0.19 \pm 0.08$ & 0.70 & $8.83 \pm 2.21$ & $0.38 \pm 0.38$ & \\
\hline & T32 Tangshan District [2] & & Yes & $\mathrm{C}$ & $2.6-3.9$ & 2.30 & $59.45 \pm 4.72$ & $50.13 \pm 3.63$ & $0.15 \pm 0.06$ & $0.94 \pm 0.06$ & $0.11 \pm 0.05$ & 0.74 & $5.63 \pm 0.75$ & $0.38 \pm 0.38$ & \\
\hline & Tientsin F13 [1] & & No & C & $3.1-5.1$ & 0.70 & $75.80 \pm 6.77$ & $42.45 \pm 3.66$ & $0.09 \pm 0.04$ & $0.93 \pm 0.08$ & $0.10 \pm 0.04$ & 0.60 & $1.63 \pm 0.35$ & $2.62 \pm 0.74$ & \\
\hline & T21 Tangshan District [2] & & No & $\mathrm{C}$ & $3.1-4.0$ & 3.10 & $59.93 \pm 3.66$ & $55.51 \pm 3.03$ & $0.20 \pm 0.08$ & $0.93 \pm 0.07$ & $0.13 \pm 0.05$ & 0.72 & $15.52 \pm 1.21$ & $0.38 \pm 0.38$ & \\
\hline & T30 Tangshan District [2] & & No & $\mathrm{C}$ & $5.0-8.0$ & 2.50 & $116.00 \pm 10.01$ & $76.76 \pm 4.78$ & $0.10 \pm 0.04$ & $0.86 \pm 0.11$ & $0.08 \pm 0.04$ & 0.65 & $14.92 \pm 1.64$ & $0.38 \pm 0.38$ & \\
\hline & T36 Tangshan District [2] & & No & $\mathrm{C}$ & $5.7-9.0$ & 2.30 & $132.75 \pm 11.07$ & $83.21 \pm 5.33$ & $0.15 \pm 0.06$ & $0.82 \pm 0.13$ & $0.13 \pm 0.06$ & 0.72 & $7.61 \pm 1.10$ & $0.38 \pm 0.38$ & \\
\hline 1977 Vrancea, Romania & 2 & $7.20 \pm 0.11$ & No & $\mathrm{C}$ & $6.5-9.0$ & 1.00 & $144.25 \pm 8.76$ & $78.03 \pm 5.47$ & $0.10 \pm 0.04$ & $0.79 \pm 0.13$ & $0.13 \pm 0.06$ & 0.55 & $3.45 \pm 1.82$ & $0.38 \pm 0.38$ & Ishihara and Perlea (1984) \\
\hline \multirow{5}{*}{$\begin{array}{l}1979 \text { Imperial Valley, } \\
\text { USA }\end{array}$} & Radio Tower B1 & $6.50 \pm 0.13$ & Yes & A & $3.0-5.5$ & 2.01 & $74.72 \pm 8.20$ & $52.75 \pm 4.53$ & $0.18 \pm 0.02$ & $0.89 \pm 0.08$ & $0.16 \pm 0.03$ & 0.52 & $4.38 \pm 2.21$ & $0.96 \pm 0.58$ & \multirow{5}{*}{$\begin{array}{l}\text { Bennett et al. (1984); } \\
\text { Bierschwale and Stokoe (1984) }\end{array}$} \\
\hline & McKim Ranch A & & Yes & A & $1.5-4.0$ & 1.50 & $47.75 \pm 8.12$ & $35.49 \pm 4.38$ & $0.51 \pm 0.05$ & $0.91 \pm 0.05$ & $0.44 \pm 0.07$ & 0.52 & $4.61 \pm 1.48$ & $1.13 \pm 0.40$ & \\
\hline & Kornbloom B & & No & A & $2.6-5.2$ & 2.74 & $65.88 \pm 8.50$ & $54.50 \pm 4.58$ & $0.13 \pm 0.04$ & $0.91 \pm 0.07$ & $0.09 \pm 0.01$ & 0.44 & $3.65 \pm 2.48$ & $2.45 \pm 1.87$ & \\
\hline & Wildlife B & & No & B & $3.7-6.7$ & 0.90 & $98.70 \pm 10.22$ & $56.52 \pm 4.90$ & $0.17 \pm 0.05$ & $0.86 \pm 0.09$ & $0.13 \pm 0.04$ & 0.40 & $6.45 \pm 3.83$ & $1.50 \pm 1.00$ & \\
\hline & Radio Tower B2 & & No & B & $2.0-3.0$ & 2.01 & $41.47 \pm 3.65$ & $36.66 \pm 3.71$ & $0.16 \pm 0.02$ & $0.95 \pm 0.05$ & $0.12 \pm 0.02$ & 0.40 & $8.59 \pm 5.47$ & $1.41 \pm 1.12$ & \\
\hline \multirow[t]{4}{*}{1980 Mexicali, Mexico } & Delta Site 2 & $6.20 \pm 0.14$ & Yes & B & $2.2-3.2$ & 2.20 & $44.20 \pm 3.36$ & $39.30 \pm 4.19$ & $0.19 \pm 0.05$ & $0.94 \pm 0.05$ & 0.14 & 0.90 & $7.28 \pm 1.33$ & $0.04 \pm 0.01$ & \multirow{4}{*}{$\begin{array}{l}\text { Diaz-Rodngues } \\
(1983,1984) ; \\
\text { Anderson (1982) }\end{array}$} \\
\hline & Delta Site 3 & & Yes & B & $2.0-3.8$ & 2.00 & $48.20 \pm 5.60$ & $39.37 \pm 4.46$ & $0.19 \pm 0.05$ & $0.93 \pm 0.06$ & 0.15 & 0.65 & $3.14 \pm 0.56$ & $0.78 \pm 0.20$ & \\
\hline & Delta Site $3 p$ & & Yes & B & $2.2-3.8$ & 2.20 & $49.60 \pm 5.04$ & $41.75 \pm 4.40$ & $0.19 \pm 0.05$ & $0.93 \pm 0.06$ & 0.14 & 0.58 & $3.19 \pm 0.96$ & $0.93 \pm 0.31$ & \\
\hline & Delta Site 4 & & Yes & B & $2.0-2.6$ & 2.00 & $37.40 \pm 2.29$ & $34.46 \pm 4.08$ & $0.19 \pm 0.05$ & $0.95 \pm 0.05$ & 0.13 & 0.53 & $5.28 \pm 0.46$ & $0.81 \pm 0.10$ & \\
\hline
\end{tabular}


Table 1. (Continued.)

\begin{tabular}{|c|c|c|c|c|c|c|c|c|c|c|c|c|c|c|c|}
\hline Earthquake & Site & $M_{w}$ & Liquefied? & $\begin{array}{l}\text { Data } \\
\text { class }\end{array}$ & $\begin{array}{l}\text { Crit. } \\
\text { depth } \\
\text { range } \\
(\mathrm{m})\end{array}$ & $\begin{array}{l}\text { Depth } \\
\text { to } \\
\text { GWT } \\
\text { (m) }\end{array}$ & $\begin{array}{c}\sigma_{\mathrm{vo}} \\
(\mathrm{kPa})\end{array}$ & $\begin{array}{c}\sigma_{\mathrm{vo}}^{\prime} \\
(\mathrm{kPa})\end{array}$ & $\begin{array}{c}a_{\max } \\
(\mathrm{g})\end{array}$ & $r_{d}$ & CSR & $c$ & $\begin{array}{c}q_{c 1} \\
(\mathrm{MPa})\end{array}$ & $\begin{array}{c}R_{f} \\
(\%)\end{array}$ & Reference \\
\hline \multirow{6}{*}{$\begin{array}{l}1981 \text { Westmorland, } \\
\text { USA }\end{array}$} & Delta Site 1 & & No & В & $4.8-5.3$ & 2.30 & $86.30 \pm 2.54$ & $59.32 \pm 4.33$ & $0.19 \pm 0.05$ & $0.86 \pm 0.09$ & 0.16 & 0.43 & $4.68 \pm 0.01$ & $1.96 \pm 1.12$ & \multirow{6}{*}{$\begin{array}{l}\text { Bennett et al. (1984); } \\
\text { Bierschwale and Stokoe } \\
\text { (1984); Youd and Wieczorek } \\
\text { (1984) }\end{array}$} \\
\hline & Wildlife B & $5.90 \pm 0.15$ & Yes & В & $2.7-6.7$ & 0.91 & $89.31 \pm 13.34$ & $51.93 \pm 5.94$ & $0.23 \pm 0.02$ & $0.86 \pm 0.09$ & $0.24 \pm 0.06$ & 0.43 & $6.80 \pm 3.13$ & $1.38 \pm 0.77$ & \\
\hline & Kornbloom B & & Yes & В & $2.8-5.8$ & 2.74 & $73.48 \pm 9.75$ & $58.18 \pm 4.86$ & $0.19 \pm 0.03$ & $0.88 \pm 0.08$ & $0.14 \pm 0.03$ & 0.40 & $3.20 \pm 1.88$ & $2.78 \pm 1.79$ & \\
\hline & Radio Tower B1 & & Yes & A & $2.0-5.5$ & 2.00 & $72.50 \pm 7.71$ & $50.43 \pm 4.92$ & $0.17 \pm 0.02$ & $0.89 \pm 0.08$ & $0.14 \pm 0.02$ & 0.52 & $4.61 \pm 1.99$ & $0.88 \pm 0.42$ & \\
\hline & McKim Ranch A & & No & B & $1.5-5.2$ & 1.50 & $57.30 \pm 11.09$ & $39.15 \pm 5.56$ & $0.09 \pm 0.02$ & $0.92 \pm 0.06$ & $0.08 \pm 0.02$ & 0.50 & $5.29 \pm 1.35$ & $1.13 \pm 0.32$ & \\
\hline & Radio Tower B2 & & No & A & $2.0-3.0$ & 2.01 & $40.98 \pm 3.33$ & $36.17 \pm 4.17$ & $0.16 \pm 0.02$ & $0.94 \pm 0.05$ & $0.12 \pm 0.02$ & 0.40 & $9.52 \pm 4.57$ & $1.36 \pm 0.73$ & \\
\hline \multirow{3}{*}{$\begin{array}{l}1983 \text { Nihonkai-Chubu, } \\
\text { Japan }\end{array}$} & Akita A & $7.70 \pm 0.10$ & Yes & $\mathrm{C}$ & $0.8-6.5$ & 0.78 & $64.16 \pm 18.49$ & $37.48 \pm 6.60$ & $0.17 \pm 0.05$ & $0.93 \pm 0.07$ & $0.18 \pm 0.08$ & 0.40 & $5.44 \pm 3.38$ & $2.01 \pm 2.66$ & \multirow{3}{*}{ Farrar (1990) } \\
\hline & Akita B & & Yes & B & $3.3-6.7$ & 1.03 & $91.91 \pm 12.97$ & $52.96 \pm 5.30$ & $0.17 \pm 0.05$ & $0.89 \pm 0.09$ & $0.17 \pm 0.06$ & 0.52 & $3.93 \pm 1.84$ & $1.05 \pm 1.28$ & \\
\hline & Akita C & & No & B & $2.0-4.0$ & 2.40 & $49.80 \pm 6.59$ & $43.91 \pm 3.31$ & $0.17 \pm 0.05$ & $0.94 \pm 0.06$ & $0.12 \pm 0.04$ & 0.48 & $4.04 \pm 0.96$ & $1.77 \pm 0.91$ & \\
\hline \multirow[t]{4}{*}{1983 Borah Peak, USA } & Pence Ranch [1] & $6.90 \pm 0.12$ & Yes & B & $1.5-4.0$ & 1.55 & $49.75 \pm 8.26$ & $37.98 \pm 3.92$ & $0.30 \pm 0.06$ & $0.93 \pm 0.05$ & $0.24 \pm 0.07$ & 0.43 & $7.54 \pm 2.24$ & $1.38 \pm 0.76$ & \multirow{4}{*}{ [1] Andrus et al. (1991) } \\
\hline & $\begin{array}{l}\text { Whiskey Springs } \\
\text { Site } 1[2]\end{array}$ & & Yes & B & $1.6-3.2$ & 0.80 & $44.80 \pm 5.38$ & $29.10 \pm 3.13$ & $0.50 \pm 0.10$ & $0.93 \pm 0.05$ & $0.46 \pm 0.12$ & 0.35 & $8.87 \pm 5.04$ & $1.83 \pm 1.89$ & \\
\hline & $\begin{array}{l}\text { Whiskey Springs } \\
\text { Site } 2[2]\end{array}$ & & Yes & B & $2.4-4.3$ & 2.40 & $59.33 \pm 6.44$ & $50.01 \pm 3.57$ & $0.50 \pm 0.10$ & $0.89 \pm 0.06$ & $0.34 \pm 0.09$ & 0.32 & $6.60 \pm 3.03$ & $3.90 \pm 3.11$ & \\
\hline & $\begin{array}{l}\text { Whiskey Springs } \\
\text { Site } 3[2]\end{array}$ & & Yes & B & $6.8-7.8$ & 6.80 & $125.45 \pm 5.49$ & $120.45 \pm 5.03$ & $0.50 \pm 0.10$ & $0.70 \pm 0.13$ & $0.24 \pm 0.07$ & 0.33 & $7.80 \pm 2.07$ & $2.58 \pm 1.65$ & \\
\hline \multirow{17}{*}{$\begin{array}{l}1987 \text { Edgecumbe, New } \\
\text { Zealand }\end{array}$} & Robinson Farm E. & $6.60 \pm 0.13$ & Yes & B & $2.0-5.5$ & 0.76 & $57.67 \pm 9.26$ & $28.03 \pm 4.29$ & $0.44 \pm 0.09$ & $0.88 \pm 0.07$ & $0.51 \pm 0.16$ & 0.60 & $10.54 \pm 4.38$ & $0.37 \pm 0.19$ & \multirow{17}{*}{$\begin{array}{l}\text { Christensen (1995); } \\
\text { Zhao et al. (1997) }\end{array}$} \\
\hline & Robinson Farm W. & & Yes & $\mathrm{C}$ & $1.0-2.8$ & 0.61 & $28.84 \pm 4.75$ & $16.19 \pm 3.13$ & $0.44 \pm 0.13$ & $0.95 \pm 0.04$ & $0.48 \pm 0.19$ & 0.73 & $13.84 \pm 1.97$ & $0.10 \pm 0.00$ & \\
\hline & Gordon Farm1 & & Yes & B & $1.2-2.4$ & 0.47 & $41.38 \pm 7.89$ & $19.50 \pm 3.82$ & $0.43 \pm 0.09$ & $0.92 \pm 0.05$ & $0.55 \pm 0.19$ & 0.53 & $8.05 \pm 2.68$ & $0.65 \pm 0.25$ & \\
\hline & Brady Farm1 & & Yes & $\mathrm{C}$ & $6.4-8.0$ & 1.65 & $117.70 \pm 5.77$ & $58.35 \pm 4.97$ & $0.40 \pm 0.12$ & $0.70 \pm 0.13$ & $0.37 \pm 0.13$ & 0.52 & $3.09 \pm 1.07$ & $0.97 \pm 0.37$ & \\
\hline & Morris Farm1 & & Yes & В & $7.0-8.5$ & 1.63 & $118.50 \pm 5.62$ & $58.46 \pm 4.98$ & $0.42 \pm 0.08$ & $0.69 \pm 0.13$ & $0.38 \pm 0.11$ & 0.58 & $10.39 \pm 1.17$ & $0.37 \pm 0.06$ & \\
\hline & Awaroa Farm & & Yes & В & $2.3-3.3$ & 1.15 & $42.25 \pm 2.90$ & $26.06 \pm 3.04$ & $0.37 \pm 0.07$ & $0.92 \pm 0.06$ & $0.36 \pm 0.09$ & 0.38 & $11.36 \pm 2.20$ & $1.10 \pm 0.25$ & \\
\hline & Keir Farm & & Yes & В & $6.5-9.5$ & 2.54 & $121.46 \pm 8.66$ & $67.90 \pm 5.23$ & $0.31 \pm 0.06$ & $0.71 \pm 0.14$ & $0.26 \pm 0.08$ & 0.43 & $8.61 \pm 1.24$ & $0.31 \pm 0.06$ & \\
\hline & James St. Loop & & Yes & B & $3.4-6.8$ & 1.15 & $77.90 \pm 9.17$ & $39.15 \pm 4.58$ & $0.28 \pm 0.06$ & $0.85 \pm 0.09$ & $0.31 \pm 0.09$ & 0.53 & $9.08 \pm 3.00$ & $0.56 \pm 0.24$ & \\
\hline & Landing Rd. Bridge & & Yes & B & $4.8-6.2$ & 1.15 & $84.10 \pm 4.63$ & $41.43 \pm 4.06$ & $0.27 \pm 0.05$ & $0.83 \pm 0.10$ & $0.30 \pm 0.08$ & 0.63 & $10.57 \pm 2.07$ & $0.32 \pm 0.07$ & \\
\hline & Whakatane Pony Club & & Yes & В & $3.6-4.6$ & 2.35 & $61.20 \pm 3.21$ & $44.03 \pm 3.33$ & $0.27 \pm 0.05$ & $0.89 \pm 0.08$ & $0.22 \pm 0.05$ & 0.88 & $8.60 \pm 1.59$ & $0.10 \pm 0.03$ & \\
\hline & Sewage Pumping Station & & Yes & B & $2.0-8.0$ & 1.29 & $76.21 \pm 15.71$ & $39.81 \pm 5.94$ & $0.26 \pm 0.05$ & $0.85 \pm 0.09$ & $0.28 \pm 0.09$ & 0.67 & $7.47 \pm 2.34$ & $0.30 \pm 0.21$ & \\
\hline & Edgecumbe Pipe Breaks & & Yes & B & $5.0-5.9$ & 2.50 & $81.98 \pm 3.41$ & $53.04 \pm 3.69$ & $0.39 \pm 0.08$ & $0.81 \pm 0.10$ & $0.32 \pm 0.08$ & 0.40 & $7.77 \pm 1.57$ & $0.39 \pm 0.12$ & \\
\hline & Gordon Farm2 & & No & B & $1.7-1.9$ & 0.90 & $27.00 \pm 1.01$ & $18.17 \pm 2.77$ & $0.37 \pm 0.07$ & $0.95 \pm 0.04$ & $0.34 \pm 0.09$ & 0.50 & $21.57 \pm 3.25$ & $0.50 \pm 0.26$ & \\
\hline & Brady Farm4 & & No & В & $3.4-5.0$ & 1.53 & $63.57 \pm 4.59$ & $37.38 \pm 3.53$ & $0.40 \pm 0.12$ & $0.86 \pm 0.08$ & $0.38 \pm 0.13$ & 0.56 & $13.24 \pm 2.09$ & $0.41 \pm 0.13$ & \\
\hline & Morris Farm3 & & No & B & $5.2-6.6$ & 2.10 & $89.35 \pm 4.57$ & $52.07 \pm 3.99$ & $0.41 \pm 0.12$ & $0.78 \pm 0.11$ & $0.36 \pm 0.12$ & 0.65 & $12.23 \pm 2.08$ & $0.31 \pm 0.12$ & \\
\hline & Whakatane Hospital & & No & B & $4.4-5.0$ & 4.40 & $68.45 \pm 3.23$ & $65.51 \pm 3.90$ & $0.26 \pm 0.05$ & $0.87 \pm 0.09$ & $0.15 \pm 0.04$ & 0.50 & $17.05 \pm 2.25$ & $0.49 \pm 0.09$ & \\
\hline & Whakatane Board Mill & & No & B & $7.0-8.0$ & 1.44 & $114.81 \pm 4.76$ & $55.36 \pm 4.85$ & $0.27 \pm 0.08$ & $0.74 \pm 0.13$ & $0.27 \pm 0.10$ & 0.63 & $10.73 \pm 2.94$ & $0.43 \pm 0.17$ & \\
\hline $\begin{array}{l}1987 \text { Elmore Ranch, } \\
\text { USA }\end{array}$ & Wildlife B & $6.20 \pm 0.14$ & No & B & $3.7-6.7$ & 0.90 & $98.70 \pm 10.22$ & $56.52 \pm 4.90$ & $0.17 \pm 0.05$ & $0.85 \pm 0.09$ & $0.16 \pm 0.05$ & 0.40 & $6.45 \pm 3.83$ & $1.50 \pm 1.00$ & $\begin{array}{l}\text { Bennett et al. (1984); } \\
\text { Bierschwale and Stokoe (1984) }\end{array}$ \\
\hline $\begin{array}{l}1987 \text { Superstition Hills, } \\
\text { USA }\end{array}$ & Wildlife B & $6.60 \pm 0.13$ & Yes & B & $3.7-6.7$ & 0.90 & $98.70 \pm 10.22$ & $56.52 \pm 4.90$ & $0.21 \pm 0.05$ & $0.85 \pm 0.09$ & $0.20 \pm 0.06$ & 0.40 & $6.45 \pm 3.83$ & $1.50 \pm 1.00$ & $\begin{array}{l}\text { Bennett et al.(1984); } \\
\text { Bierschwale and Stokoe (1984) }\end{array}$ \\
\hline
\end{tabular}




\begin{tabular}{|c|c|c|c|c|c|c|c|c|c|c|c|c|c|c|c|}
\hline Earthquake & Site & $M_{w}$ & Liquefied? & $\begin{array}{l}\text { Data } \\
\text { class }\end{array}$ & $\begin{array}{l}\text { Crit. } \\
\text { depth } \\
\text { range } \\
(\mathrm{m})\end{array}$ & $\begin{array}{c}\text { Depth } \\
\text { to } \\
\text { GWT } \\
\text { (m) }\end{array}$ & $\begin{array}{c}\sigma_{\mathrm{vo}} \\
(\mathrm{kPa})\end{array}$ & $\begin{array}{c}\sigma_{\mathrm{vo}}^{\prime} \\
(\mathrm{kPa})\end{array}$ & $\begin{array}{l}a_{\max } \\
(\mathrm{g})\end{array}$ & $r_{d}$ & CSR & $c$ & $\begin{array}{c}q_{c 1} \\
(\mathrm{MPa})\end{array}$ & $\begin{array}{c}R_{f} \\
(\%)\end{array}$ & Reference \\
\hline \multirow{36}{*}{$\begin{array}{l}1989 \text { Loma Prieta, } \\
\text { USA }\end{array}$} & SFOBB-1 [1] & $7.00 \pm 0.12$ & Yes & A & $6.25-7.0$ & 2.99 & $127.53 \pm 4.03$ & $90.64 \pm 3.90$ & $0.28 \pm 0.01$ & $0.79 \pm 0.01$ & $0.17 \pm 0.01$ & 0.66 & $5.63 \pm 0.73$ & $0.66 \pm 0.13$ & \multirow{36}{*}{$\begin{array}{l}\text { [1] Mitchell et al. (1994); } \\
\text { Kayen et al. (1998); } \\
\text { [2] Boulanger et al. (1995); } \\
\text { Woodward-Clyde (1990); } \\
\text { Rutherford Chekene (1987, } \\
\text { 1988); } \\
\text { [3] DeAlba et al. (1994); } \\
\text { Rollins et al. (1994); } \\
\text { [4] Holzer et al. (1994); } \\
\text { [5] Bennett and Tinsley } \\
\text { (1995); } \\
\text { Toprak et al. (1999) }\end{array}$} \\
\hline & SFOBB-2 [1] & & Yes & A & $6.5-8.5$ & 2.99 & $141.03 \pm 7.74$ & $96.79 \pm 4.72$ & $0.28 \pm 0.01$ & $0.76 \pm 0.02$ & $0.18 \pm 0.01$ & 0.55 & $8.84 \pm 1.95$ & $0.55 \pm 0.23$ & \\
\hline & POO7-2 [1] & & Yes & B & $5.5-6.8$ & 2.30 & $111.18 \pm 13.02$ & $73.41 \pm 5.50$ & $0.28 \pm 0.03$ & $0.81 \pm 0.11$ & $0.22 \pm 0.05$ & 0.70 & $7.09 \pm 0.84$ & $0.45 \pm 0.06$ & \\
\hline & POO7-3 [1] & & Yes & B & $7.1-8.1$ & 2.30 & $137.50 \pm 4.95$ & $85.51 \pm 4.35$ & $0.28 \pm 0.03$ & $0.75 \pm 0.13$ & $0.22 \pm 0.05$ & 0.67 & $10.84 \pm 1.20$ & $0.25 \pm 0.05$ & \\
\hline & POR-2 [1] & & Yes & B & $5.3-6.7$ & 2.40 & $114.15 \pm 7.95$ & $74.42 \pm 4.17$ & $0.16 \pm 0.03$ & $0.82 \pm 0.11$ & $0.13 \pm 0.03$ & 0.74 & $2.66 \pm 0.76$ & $0.63 \pm 0.20$ & \\
\hline & POR-3 [1] & & Yes & B & $5.0-7.0$ & 2.40 & $106.80 \pm 6.97$ & $71.48 \pm 4.01$ & $0.16 \pm 0.03$ & $0.84 \pm 0.11$ & $0.13 \pm 0.03$ & 0.78 & $2.64 \pm 1.15$ & $0.48 \pm 0.23$ & \\
\hline & POR-4 [1] & & Yes & B & $6.0-7.0$ & 2.40 & $116.30 \pm 4.48$ & $76.08 \pm 3.81$ & $0.16 \pm 0.03$ & $0.82 \pm 0.03$ & $0.13 \pm 0.03$ & 0.80 & $2.88 \pm 0.59$ & $0.43 \pm 0.10$ & \\
\hline & Marine Lab C4 [2] & & Yes & A & $5.2-5.8$ & 2.50 & $95.75 \pm 3.31$ & $66.32 \pm 3.19$ & $0.25 \pm 0.03$ & $0.84 \pm 0.10$ & $0.20 \pm 0.03$ & 0.78 & $2.92 \pm 0.58$ & $0.51 \pm 0.16$ & \\
\hline & Marine Lab UC-7 [2] & & Yes & B & $7.6-9.8$ & 2.00 & $148.55 \pm 10.20$ & $86.75 \pm 5.68$ & $0.25 \pm 0.03$ & $0.73 \pm 0.14$ & $0.20 \pm 0.05$ & 0.55 & $4.90 \pm 1.53$ & $1.20 \pm 0.57$ & \\
\hline & Sandholdt Rd. UC-4 [2] & & Yes & A & $2.4-4.6$ & 2.70 & $56.40 \pm 7.28$ & $48.55 \pm 2.99$ & $0.25 \pm 0.03$ & $0.99 \pm 0.01$ & $0.23 \pm 0.03$ & 0.60 & $11.66 \pm 8.81$ & $0.44 \pm 0.46$ & \\
\hline & $\begin{array}{l}\text { Moss Landing S.B. } \\
\text { UC-14 [2] }\end{array}$ & & Yes & A & $2.4-4.0$ & 2.40 & $52.40 \pm 5.60$ & $44.55 \pm 3.86$ & $0.25 \pm 0.03$ & $0.95 \pm 0.01$ & $0.21 \pm 0.03$ & 0.65 & $7.91 \pm 1.15$ & $0.55 \pm 0.10$ & \\
\hline & $\begin{array}{l}\text { Woodward Marine } \\
\text { UC-11 [2] }\end{array}$ & & Yes & B & $2.5-3.4$ & 2.50 & $46.65 \pm 3.60$ & $43.22 \pm 3.88$ & $0.25 \pm 0.03$ & $0.99 \pm 0.01$ & $0.20 \pm 0.04$ & 0.64 & $9.40 \pm 1.71$ & $0.48 \pm 0.10$ & \\
\hline & $\begin{array}{l}\text { Habor Office } \\
\text { UC-12\&13 [2] }\end{array}$ & & Yes & B & $2.9-4.7$ & 1.90 & $66.50 \pm 6.14$ & $47.86 \pm 4.24$ & $0.25 \pm 0.08$ & $0.91 \pm 0.07$ & $0.20 \pm 0.07$ & 0.56 & $8.98 \pm 5.23$ & $0.58 \pm 0.36$ & \\
\hline & T.I. Naval Station [3] & & Yes & B & $3.5-7.0$ & 1.50 & $97.43 \pm 11.60$ & $60.64 \pm 4.67$ & $0.16 \pm 0.03$ & $0.87 \pm 0.10$ & $0.14 \pm 0.04$ & 0.60 & $5.05 \pm 1.91$ & $0.85 \pm 0.50$ & \\
\hline & Farris Farm Site [4] & & Yes & A & $6.0-7.0$ & 4.50 & $106.75 \pm 4.50$ & $87.13 \pm 3.87$ & $0.31 \pm 0.08$ & $0.90 \pm 0.02$ & $0.28 \pm 0.05$ & 0.67 & $4.44 \pm 0.52$ & $0.71 \pm 0.10$ & \\
\hline & Miller Farm CMF 8 [5] & & Yes & A & $6.8-8.0$ & 4.91 & $123.42 \pm 5.29$ & $98.99 \pm 4.16$ & $0.30 \pm 0.07$ & $0.73 \pm 0.01$ & $0.25 \pm 0.03$ & 0.81 & $4.83 \pm 0.94$ & $0.25 \pm 0.20$ & \\
\hline & Miller Farm CMF 10 [5] & & Yes & A & $7.0-9.7$ & 3.00 & $155.35 \pm 9.52$ & $99.92 \pm 5.36$ & $0.30 \pm 0.07$ & $0.88 \pm 0.02$ & $0.37 \pm 0.06$ & 0.45 & $4.80 \pm 2.41$ & $1.93 \pm 0.99$ & \\
\hline & Miller Farm CMF 5 [5] & & Yes & A & $5.5-8.5$ & 4.70 & $122.40 \pm 10.47$ & $99.84 \pm 5.18$ & $0.30 \pm 0.07$ & $0.77 \pm 0.12$ & $0.29 \pm 0.04$ & 0.63 & $7.13 \pm 1.57$ & $0.49 \pm 0.20$ & \\
\hline & Miller Farm CMF 3 [5] & & Yes & A & $5.75-7.5$ & 3.00 & $103.55 \pm 6.74$ & $95.70 \pm 4.46$ & $0.30 \pm 0.07$ & $0.83 \pm 0.02$ & $0.26 \pm 0.04$ & 0.71 & $3.27 \pm 1.44$ & $0.72 \pm 0.44$ & \\
\hline & Model Airport 18 [5] & & Yes & B & $3.7-4.5$ & 2.40 & $70.70 \pm 3.28$ & $54.02 \pm 2.90$ & $0.29 \pm 0.07$ & $0.89 \pm 0.08$ & $0.22 \pm 0.06$ & 0.72 & $8.93 \pm 1.45$ & $0.35 \pm 0.09$ & \\
\hline & Model Airport 21 [5] & & Yes & B & $3.4-4.7$ & 2.40 & $69.75 \pm 4.61$ & $53.56 \pm 3.07$ & $0.29 \pm 0.07$ & $0.89 \pm 0.08$ & $0.22 \pm 0.06$ & 0.74 & $8.38 \pm 2.54$ & $0.30 \pm 0.11$ & \\
\hline & Farris $58[5]$ & & Yes & B & $7.4-8.0$ & 4.80 & $131.90 \pm 4.16$ & $103.45 \pm 4.18$ & $0.31 \pm 0.08$ & $0.74 \pm 0.13$ & $0.19 \pm 0.06$ & 0.67 & $8.54 \pm 0.35$ & $0.48 \pm 0.02$ & \\
\hline & Farris $61[5]$ & & Yes & B & $6.0-7.3$ & 4.20 & $110.43 \pm 5.15$ & $86.39 \pm 3.92$ & $0.31 \pm 0.08$ & $0.78 \pm 0.12$ & $0.20 \pm 0.06$ & 0.64 & $4.27 \pm 0.58$ & $0.81 \pm 0.12$ & \\
\hline & Granite Const. 123 [5] & & Yes & B & $7.2-7.8$ & 5.00 & $127.50 \pm 4.15$ & $102.98 \pm 4.17$ & $0.31 \pm 0.08$ & $0.75 \pm 0.13$ & $0.18 \pm 0.06$ & 0.73 & $4.36 \pm 0.28$ & $0.50 \pm 0.16$ & \\
\hline & Jefferson $121[5]$ & & Yes & B & $6.5-7.75$ & 3.40 & $126.88 \pm 5.16$ & $90.33 \pm 4.14$ & $0.18 \pm 0.05$ & $0.79 \pm 0.12$ & $0.12 \pm 0.04$ & 0.71 & $6.10 \pm 0.87$ & $0.45 \pm 0.08$ & \\
\hline & Jefferson $141[5]$ & & Yes & B & $3.1-4.5$ & 2.10 & $66.95 \pm 4.82$ & $50.27 \pm 3.20$ & $0.18 \pm 0.05$ & $0.91 \pm 0.07$ & $0.13 \pm 0.04$ & 0.70 & $3.02 \pm 0.75$ & $0.83 \pm 0.26$ & \\
\hline & Jefferson $148[5]$ & & Yes & B & $7.0-7.9$ & 3.00 & $137.78 \pm 4.57$ & $94.12 \pm 4.22$ & $0.18 \pm 0.04$ & $0.78 \pm 0.13$ & $0.12 \pm 0.04$ & 0.72 & $7.20 \pm 1.81$ & $0.38 \pm 0.11$ & \\
\hline & Jefferson Ranch 32 [5] & & Yes & B & $2.3-3.1$ & 1.80 & $45.90 \pm 2.98$ & $37.07 \pm 2.55$ & $0.17 \pm 0.04$ & $0.95 \pm 0.05$ & $0.13 \pm 0.03$ & 0.79 & $5.22 \pm 0.77$ & $0.31 \pm 0.05$ & \\
\hline & Kett $74[5]$ & & Yes & B & $2.3-3.1$ & 1.50 & $48.15 \pm 3.01$ & $36.38 \pm 2.55$ & $0.32 \pm 0.08$ & $0.93 \pm 0.05$ & $0.26 \pm 0.07$ & 0.46 & $8.08 \pm 0.88$ & $1.20 \pm 0.31$ & \\
\hline & Leonardini 39 [5] & & Yes & B & $2.3-4.7$ & 1.90 & $60.80 \pm 7.82$ & $45.10 \pm 3.58$ & $0.17 \pm 0.04$ & $0.92 \pm 0.07$ & $0.14 \pm 0.04$ & 0.87 & $6.07 \pm 1.88$ & $0.16 \pm 0.05$ & \\
\hline & Leonardini $51[5]$ & & Yes & B & $3.1-3.7$ & 1.80 & $59.20 \pm 2.61$ & $43.50 \pm 2.63$ & $0.17 \pm 0.04$ & $0.93 \pm 0.07$ & $0.14 \pm 0.04$ & 0.81 & $2.39 \pm 0.32$ & $0.48 \pm 0.08$ & \\
\hline & Leonardini 53 [5] & & Yes & B & $2.7-3.6$ & 2.10 & $55.13 \pm 3.41$ & $44.82 \pm 2.73$ & $0.17 \pm 0.04$ & $0.93 \pm 0.06$ & $0.13 \pm 0.03$ & 0.78 & $6.65 \pm 0.82$ & $0.28 \pm 0.11$ & \\
\hline & Marinovich 65 [5] & & Yes & B & $6.8-9.4$ & 5.60 & $150.90 \pm 12.42$ & $121.47 \pm 6.07$ & $0.28 \pm 0.07$ & $0.95 \pm 0.09$ & $0.21 \pm 0.06$ & 0.65 & $6.33 \pm 0.48$ & $0.67 \pm 0.10$ & \\
\hline & Radovich 99 [5] & & Yes & B & $4.75-6.9$ & 4.10 & $79.38 \pm 4.42$ & $72.26 \pm 3.54$ & $0.28 \pm 0.07$ & $0.95 \pm 0.09$ & $0.19 \pm 0.05$ & 0.62 & $6.37 \pm 0.93$ & $0.74 \pm 0.15$ & \\
\hline & Sea Mist 31 [5] & & Yes & B & $2.8-3.7$ & 0.80 & $60.33 \pm 3.45$ & $36.29 \pm 2.80$ & $0.17 \pm 0.04$ & $0.95 \pm 0.09$ & $0.18 \pm 0.05$ & 0.76 & $2.67 \pm 0.79$ & $0.53 \pm 0.19$ & \\
\hline & Silliman 68 [5] & & Yes & B & $4.7-7.1$ & 3.50 & $103.37 \pm 8.23$ & $79.83 \pm 4.28$ & $0.28 \pm 0.07$ & $0.95 \pm 0.09$ & $0.22 \pm 0.06$ & 0.64 & $5.56 \pm 0.35$ & $0.69 \pm 0.05$ & \\
\hline
\end{tabular}


Table 1. (Continued.)

\begin{tabular}{|c|c|c|c|c|c|c|c|c|c|c|c|c|c|c|c|}
\hline Earthquake & Site & $M_{w}$ & Liquefied? & $\begin{array}{l}\text { Data } \\
\text { class }\end{array}$ & $\begin{array}{l}\text { Crit. } \\
\text { depth } \\
\text { range } \\
(\mathrm{m})\end{array}$ & $\begin{array}{l}\text { Depth } \\
\text { to } \\
\text { GWT } \\
(\mathrm{m})\end{array}$ & $\begin{array}{c}\sigma_{\mathrm{vo}} \\
(\mathrm{kPa})\end{array}$ & $\begin{array}{c}\sigma_{\mathrm{vo}}^{\prime} \\
(\mathrm{kPa})\end{array}$ & $\begin{array}{c}a_{\max } \\
(\mathrm{g})\end{array}$ & $r_{d}$ & CSR & $c$ & $\begin{array}{c}q_{c 1} \\
(\mathrm{MPa})\end{array}$ & $\begin{array}{c}R_{f} \\
(\%) \\
\end{array}$ & Reference \\
\hline & SP Bridge 48 [5] & & Yes & B & $6.0-7.5$ & 5.30 & $114.38 \pm 6.04$ & $100.15 \pm 4.38$ & $0.30 \pm 0.08$ & $0.95 \pm 0.09$ & $0.21 \pm 0.06$ & 0.61 & $3.95 \pm 0.73$ & $0.95 \pm 0.19$ & \\
\hline & $\begin{array}{l}\text { Alameda Bay } \\
\text { Farm Is. [1] }\end{array}$ & & No & A & $5.0-6.0$ & 2.50 & $103.75 \pm 4.23$ & $74.32 \pm 3.56$ & $0.24 \pm 0.02$ & $0.95 \pm 0.09$ & $0.16 \pm 0.03$ & 0.34 & $7.85 \pm 2.98$ & $2.15 \pm 0.89$ & \\
\hline & MBARI3 RC-6 [2] & & No & A & $3.0-4.5$ & 2.60 & $64.03 \pm 5.31$ & $52.74 \pm 3.05$ & $0.25 \pm 0.03$ & $0.91 \pm 0.07$ & $0.18 \pm 0.03$ & 0.74 & $21.48 \pm 1.39$ & $0.21 \pm 0.06$ & \\
\hline & MBARI3 RC-7 [2] & & No & A & $4.0-5.0$ & 3.70 & $74.80 \pm 4.19$ & $66.95 \pm 3.24$ & $0.25 \pm 0.03$ & $0.88 \pm 0.08$ & $0.16 \pm 0.02$ & 0.70 & $12.35 \pm 0.81$ & $0.30 \pm 0.06$ & \\
\hline & Sandholdt Rd. UC2 [2] & & No & A & $3.0-4.5$ & 2.70 & $61.20 \pm 5.40$ & $50.90 \pm 3.51$ & $0.25 \pm 0.03$ & $0.91 \pm 0.07$ & $0.18 \pm 0.03$ & 0.65 & $25.55 \pm 7.61$ & $0.30 \pm 0.10$ & \\
\hline & General Fish CPT-6 [2] & & No & A & $2.2-3.2$ & 1.70 & $48.90 \pm 3.79$ & $39.09 \pm 3.74$ & $0.25 \pm 0.03$ & $0.94 \pm 0.05$ & $0.19 \pm 0.03$ & 0.70 & $18.06 \pm 2.78$ & $0.32 \pm 0.06$ & \\
\hline & MBARI4 CPT-1 [2] & & No & A & $2.3-3.5$ & 1.90 & $48.08 \pm 4.46$ & $38.27 \pm 3.28$ & $0.25 \pm 0.03$ & $0.93 \pm 0.06$ & $0.19 \pm 0.03$ & 0.70 & $18.79 \pm 1.99$ & $0.28 \pm 0.06$ & \\
\hline & Sandholdt Rd. UC-6 [2] & & No & A & $6.2-7.0$ & 2.70 & $123.90 \pm 3.87$ & $85.64 \pm 4.26$ & $0.25 \pm 0.03$ & $0.80 \pm 0.12$ & $0.19 \pm 0.03$ & 0.70 & $20.99 \pm 0.68$ & $0.30 \pm 0.05$ & \\
\hline & $\begin{array}{l}\text { Moss Landing } \\
\text { S. B.18 [2] }\end{array}$ & & No & A & $2.4-3.4$ & 2.40 & $48.40 \pm 4.08$ & $43.50 \pm 3.32$ & $0.25 \pm 0.03$ & $0.93 \pm 0.06$ & $0.17 \pm 0.03$ & 0.72 & $18.94 \pm 1.38$ & $0.27 \pm 0.05$ & \\
\hline & Leonardini 37 [5] & & No & B & $2.9-6.1$ & 2.50 & $78.00 \pm 10.38$ & $58.38 \pm 4.39$ & $0.17 \pm 0.04$ & $0.89 \pm 0.08$ & $0.13 \pm 0.04$ & 0.74 & $5.81 \pm 1.34$ & $0.35 \pm 0.09$ & \\
\hline & Leonardini 52a [5] & & No & B & $3.8-4.5$ & 2.70 & $72.83 \pm 3.14$ & $58.60 \pm 2.94$ & $0.17 \pm 0.04$ & $0.90 \pm 0.08$ & $0.12 \pm 0.03$ & 0.60 & $3.82 \pm 1.07$ & $1.17 \pm 0.67$ & \\
\hline & Matella 111 [5] & & No & B & $1.7-5.1$ & 1.70 & $60.18 \pm 11.15$ & $43.50 \pm 4.29$ & $0.15 \pm 0.04$ & $0.93 \pm 0.07$ & $0.12 \pm 0.04$ & 0.71 & $5.16 \pm 0.98$ & $0.47 \pm 0.10$ & \\
\hline & McGowan Farm 136 [5] & & No & B & $2.4-3.1$ & 2.40 & $46.36 \pm 2.99$ & $42.92 \pm 2.74$ & $0.26 \pm 0.07$ & $0.94 \pm 0.05$ & $0.18 \pm 0.05$ & 0.57 & $6.00 \pm 0.58$ & $1.07 \pm 0.12$ & \\
\hline & Marinovich 67 [5] & & No & B & $6.2-7.0$ & 6.20 & $113.40 \pm 4.87$ & $109.48 \pm 4.57$ & $0.28 \pm 0.07$ & $0.95 \pm 0.09$ & $0.18 \pm 0.05$ & 0.55 & $14.21 \pm 1.03$ & $0.70 \pm 0.06$ & \\
\hline & Radovich 98 [5] & & No & B & $5.1-8.75$ & 3.50 & $124.54 \pm 12.30$ & $90.94 \pm 5.53$ & $0.28 \pm 0.07$ & $0.95 \pm 0.09$ & $0.24 \pm 0.07$ & 0.60 & $8.33 \pm 1.74$ & $0.68 \pm 0.30$ & \\
\hline & $\begin{array}{l}\text { Salinas River } \\
\text { Bridge } 117 \text { [5] }\end{array}$ & & No & B & $6.4-7.4$ & 6.40 & $113.97 \pm 5.29$ & $109.97 \pm 4.71$ & $0.12 \pm 0.03$ & $0.95 \pm 0.09$ & $0.08 \pm 0.02$ & 0.46 & $5.31 \pm 0.79$ & $1.64 \pm 0.39$ & \\
\hline & Tanimura $105[9]$ & & No & B & $4.2-6.8$ & 4.20 & $92.29 \pm 8.88$ & $79.54 \pm 4.35$ & $0.15 \pm 0.04$ & $0.95 \pm 0.09$ & $0.11 \pm 0.03$ & 0.75 & $4.56 \pm 0.41$ & $0.41 \pm 0.05$ & \\
\hline $\begin{array}{l}1994 \text { Northridge, } \\
\text { USA }\end{array}$ & $\begin{array}{l}\text { Balboa Blvd. Unit C }[1] \\
6.70 \pm 0.13\end{array}$ & & Yes & A & $8.3-9.8$ & 7.19 & $162.74 \pm 6.91$ & $144.99 \pm 5.59$ & $0.69 \pm 0.06$ & $0.54 \pm 0.15$ & $0.36 \pm 0.04$ & 0.33 & $6.43 \pm 3.63$ & $2.58 \pm 1.62$ & $\begin{array}{l}\text { [1] Bennett et al. (1998); } \\
\text { Holzer et al. (1999); } \\
\text { [2] Abdel-Haq and Hryciw } \\
\text { (1998) }\end{array}$ \\
\hline \multirow{11}{*}{$\begin{array}{l}1995 \text { Hyogoken-Nanbu, } \\
\text { Japan }\end{array}$} & Malden St. Unit D [1] & & Yes & B & $9.2-10.7$ & 3.90 & $169.80 \pm 6.41$ & $110.45 \pm 5.45$ & $0.51 \pm 0.06$ & $0.57 \pm 0.17$ & $0.29 \pm 0.09$ & 0.45 & $2.98 \pm 1.42$ & $2.36 \pm 1.28$ & \\
\hline & $\begin{array}{l}\text { Potrero Canyon } \\
\text { Unit C1 }[1]\end{array}$ & & Yes & A & $6.0-7.0$ & 3.30 & $122.67 \pm 4.51$ & $91.27 \pm 3.92$ & $0.40 \pm 0.04$ & $0.76 \pm 0.11$ & $0.25 \pm 0.04$ & 0.50 & $6.52 \pm 2.51$ & $1.08 \pm 0.49$ & \\
\hline & Wynne Ave. Unit C1 [1] & & Yes & A & $5.8-6.5$ & 4.30 & $112,76 \pm 3.50$ & $94.85 \pm 3.38$ & $0.54 \pm 0.04$ & $0.74 \pm 0.11$ & $4.30 \pm 0.35$ & 0.42 & $8.96 \pm 5.77$ & $1.13 \pm 0.87$ & \\
\hline & Rory Lane [2] & & Yes & A & $3.0-5.0$ & 2.70 & $66.60 \pm 6.33$ & $53.85 \pm 3.66$ & $0.77 \pm 0.11$ & $0.81 \pm 0.08$ & $2.70 \pm 0.50$ & 0.45 & $4.78 \pm 0.59$ & $1.80 \pm 0.90$ & \\
\hline & Dust Management Center & $7.20 \pm 0.11$ & Yes & B & $6.0-8.0$ & 2.00 & $119.50 \pm 6.72$ & $70.45 \pm 4.92$ & $0.37 \pm 0.11$ & $0.76 \pm 0.12$ & $0.31 \pm 0.11$ & 0.64 & $7.83 \pm 2.53$ & $0.49 \pm 0.20$ & Suzuki et al. (2003) \\
\hline & Imazu Elementary School & & Yes & $\mathrm{C}$ & $8.0-12.0$ & 1.40 & $185.80 \pm 13.87$ & $101.43 \pm 7.23$ & $0.60 \pm 0.18$ & $0.56 \pm 0.17$ & $0.40 \pm 0.17$ & 0.90 & $0.80 \pm 0.19$ & $0.80 \pm 0.34$ & \\
\hline & Koyo Junior High School & & Yes & B & $6.5-7.5$ & 4.00 & $124.50 \pm 4.65$ & $95.07 \pm 3.96$ & $0.45 \pm 0.14$ & $0.74 \pm 0.12$ & $0.28 \pm 0.10$ & 0.50 & $8.03 \pm 0.54$ & $1.24 \pm 0.87$ & \\
\hline & $\begin{array}{l}\text { Kobe Customs Maya } \\
\text { Office A }\end{array}$ & & Yes & B & $4.0-9.0$ & 1.80 & $121.35 \pm 4.66$ & $75.24 \pm 3.97$ & $0.60 \pm 0.18$ & $0.72 \pm 0.11$ & $0.45 \pm 0.16$ & 0.78 & $2.93 \pm 0.34$ & $0.40 \pm 0.13$ & \\
\hline & $\begin{array}{l}\text { Kobe Customs Maya } \\
\text { Office B }\end{array}$ & & Yes & $\mathrm{B}$ & $2.0-6.0$ & 1.80 & $82.35 \pm 3.96$ & $55.86 \pm 3.12$ & $0.60 \pm 0.18$ & $0.83 \pm 0.08$ & $0.48 \pm 0.15$ & 0.54 & $6.98 \pm 0.73$ & $0.87 \pm 0.17$ & \\
\hline & Kobe Port Const. Office & & Yes & B & $3.0-5.0$ & 2.50 & $70.50 \pm 3.32$ & $55.79 \pm 2.91$ & $0.60 \pm 0.18$ & $0.85 \pm 0.08$ & $0.42 \pm 0.13$ & 0.76 & $5.99 \pm 1.15$ & $0.29 \pm 0.11$ & \\
\hline & Koyo Pump Station & & Yes & B & $5.0-6.0$ & 2.60 & $99.45 \pm 4.19$ & $71.00 \pm 3.41$ & $0.45 \pm 0.14$ & $0.81 \pm 0.10$ & $0.33 \pm 0.11$ & 0.65 & $2.38 \pm 0.57$ & $1.75 \pm 0.82$ & \\
\hline
\end{tabular}




\begin{tabular}{|c|c|c|c|c|c|c|c|c|c|c|c|c|c|c|c|}
\hline Earthquake & Site & $M_{w}$ & Liquefied? & $\begin{array}{l}\text { Data } \\
\text { class }\end{array}$ & $\begin{array}{l}\text { Crit. } \\
\text { depth } \\
\text { range } \\
(\mathrm{m})\end{array}$ & $\begin{array}{l}\text { Depth } \\
\text { to } \\
\text { GWT } \\
\text { (m) }\end{array}$ & $\begin{array}{c}\sigma_{\mathrm{vo}} \\
(\mathrm{kPa})\end{array}$ & $\begin{array}{c}\sigma_{\mathrm{vo}}^{\prime} \\
(\mathrm{kPa})\end{array}$ & $\begin{array}{c}a_{\max } \\
(\mathrm{g})\end{array}$ & $r_{d}$ & CSR & $c$ & $\begin{array}{c}q_{c 1} \\
(\mathrm{MPa})\end{array}$ & $\begin{array}{l}R_{f} \\
(\%) \\
\end{array}$ & Reference \\
\hline & Kobe Wharf Public Co. & & Yes & B & $4.0-5.5$ & 2.10 & $88.63 \pm 5.41$ & $60.33 \pm 3.41$ & $0.45 \pm 0.14$ & $0.84 \pm 0.09$ & $0.35 \pm 0.12$ & 0.65 & $6.03 \pm 0.74$ & $0.78 \pm 0.40$ & \\
\hline & Koyo Elementary School & & Yes & B & $6.5-7.0$ & 4.20 & $119.03 \pm 4.61$ & $94.01 \pm 3.91$ & $0.45 \pm 0.14$ & $0.75 \pm 0.12$ & $0.28 \pm 0.10$ & 0.54 & $2.93 \pm 1.44$ & $2.17 \pm 1.50$ & \\
\hline & Mizukasa Park & & Yes & $\mathrm{C}$ & $6.9-7.9$ & 2.00 & $138.30 \pm 5.00$ & $85.33 \pm 4.36$ & $0.65 \pm 0.20$ & $0.66 \pm 0.13$ & $0.45 \pm 0.16$ & 0.75 & $1.63 \pm 0.60$ & $0.99 \pm 0.48$ & \\
\hline & $\begin{array}{l}\text { Shiporex Kogyo Osaka } \\
\text { Factory }\end{array}$ & & Yes & B & $4.0-7.0$ & 1.50 & $93.95 \pm 6.39$ & $54.71 \pm 4.44$ & $0.40 \pm 0.12$ & $0.82 \pm 0.10$ & $0.37 \pm 0.12$ & 0.74 & $3.93 \pm 2.18$ & $0.41 \pm 0.24$ & \\
\hline & $\begin{array}{l}\text { Hamakoshienn Housing } \\
\text { Area }\end{array}$ & & Yes & B & $2.5-5.0$ & 2.00 & $67.13 \pm 8.35$ & $49.96 \pm 3.85$ & $0.50 \pm 0.15$ & $0.88 \pm 0.07$ & $0.38 \pm 0.13$ & 0.59 & $7.00 \pm 1.51$ & $0.65 \pm 0.22$ & \\
\hline & Taito Kobe Factory & & Yes & B & $3.2-4.2$ & 1.60 & $62.73 \pm 3.35$ & $42.13 \pm 3.38$ & $0.45 \pm 0.14$ & $0.89 \pm 0.07$ & $0.39 \pm 0.13$ & 0.75 & $4.85 \pm 0.86$ & $0.39 \pm 0.12$ & \\
\hline & $\begin{array}{l}\text { Tokuyama Concrete } \\
\text { Factory }\end{array}$ & & Yes & B & $4.0-4.8$ & 2.00 & $74.52 \pm 3.06$ & $50.98 \pm 3.48$ & $0.50 \pm 0.15$ & $0.85 \pm 0.08$ & $0.40 \pm 0.13$ & 0.80 & $2.55 \pm 0.88$ & $0.40 \pm 0.19$ & \\
\hline & Nisseki Kobe Oil Tank A & & Yes & B & $4.8-6.1$ & 2.40 & $99.08 \pm 4.98$ & $69.15 \pm 3.53$ & $0.60 \pm 0.18$ & $0.78 \pm 0.10$ & $0.43 \pm 0.14$ & 0.72 & $5.30 \pm 1.31$ & $0.61 \pm 0.36$ & \\
\hline & Nisseki Kobe Oil Tank B & & Yes & B & $5.0-6.0$ & 2.40 & $100.05 \pm 4.20$ & $69.64 \pm 3.42$ & $0.60 \pm 0.18$ & $0.78 \pm 0.10$ & $0.43 \pm 0.14$ & 0.70 & $6.25 \pm 1.34$ & $0.74 \pm 0.27$ & \\
\hline & New Port No. 6 Pier & & Yes & B & $3.5-5.5$ & 2.50 & $70.50 \pm 6.82$ & $55.79 \pm 3.55$ & $0.60 \pm 0.18$ & $0.85 \pm 0.08$ & $0.42 \pm 0.14$ & 0.70 & $9.47 \pm 1.60$ & $0.43 \pm 0.11$ & \\
\hline & Minatojima Junior High & & Yes & B & $4.0-4.5$ & 2.70 & $74.78 \pm 2.72$ & $59.57 \pm 2.91$ & $0.45 \pm 0.14$ & $0.86 \pm 0.08$ & $0.32 \pm 0.10$ & 0.65 & $4.71 \pm 1.35$ & $0.94 \pm 0.42$ & \\
\hline & $\begin{array}{l}\text { New Wharf Const. } \\
\text { Offices }\end{array}$ & & Yes & B & $3.2-3.8$ & 2.60 & $60.45 \pm 2.78$ & $51.62 \pm 2.78$ & $0.45 \pm 0.14$ & $0.89 \pm 0.07$ & $0.31 \pm 0.10$ & 0.64 & $3.56 \pm 0.81$ & $0.93 \pm 0.64$ & \\
\hline & Fukuzumi Park & & No & $\mathrm{C}$ & $\begin{array}{c}11.0- \\
12.5\end{array}$ & 3.10 & $200.80 \pm 8.24$ & $115.94 \pm 6.85$ & $0.65 \pm 0.20$ & $0.48 \pm 0.19$ & $0.35 \pm 0.18$ & 0.40 & $17.09 \pm 3.45$ & $1.42 \pm 0.57$ & \\
\hline & Honjyo Central Park & & No & B & $4.0-6.0$ & 2.50 & $95.00 \pm 7.25$ & $70.48 \pm 3.98$ & $0.70 \pm 0.21$ & $0.78 \pm 0.09$ & $0.48 \pm 0.16$ & 0.56 & $17.30 \pm 3.75$ & $0.60 \pm 0.25$ & \\
\hline & Kobe Art Institute & & No & B & $3.5-3.8$ & 3.00 & $64.00 \pm 2.38$ & $57.62 \pm 2.86$ & $0.50 \pm 0.15$ & $0.88 \pm 0.07$ & $0.32 \pm 0.10$ & 0.33 & $13.64 \pm 5.38$ & $1.90 \pm 1.31$ & \\
\hline & Yoshida Kogyo Factory & & No & B & $3.0-5.0$ & 3.00 & $69.00 \pm 6.87$ & $59.19 \pm 3.64$ & $0.50 \pm 0.15$ & $0.87 \pm 0.08$ & $0.33 \pm 0.11$ & 0.34 & $9.43 \pm 7.22$ & $2.71 \pm 2.73$ & \\
\hline & Shimonakajima Park & & No & B & $3.0-4.5$ & 2.00 & $63.28 \pm 3.36$ & $46.11 \pm 3.38$ & $0.65 \pm 0.20$ & $0.86 \pm 0.07$ & $0.50 \pm 0.16$ & 0.53 & $19.49 \pm 0.80$ & $0.73 \pm 0.43$ & \\
\hline & Sumiyoshi Elementary & & No & B & $2.4-3.2$ & 1.90 & $46.92 \pm 2.68$ & $38.09 \pm 3.15$ & $0.60 \pm 0.18$ & $0.91 \pm 0.06$ & $0.43 \pm 0.14$ & 0.54 & $17.35 \pm 4.20$ & $0.66 \pm 0.31$ & \\
\hline & Nagashi Park & & No & B & $1.1-1.8$ & 1.00 & $26.00 \pm 2.60$ & $21.59 \pm 2.32$ & $0.65 \pm 0.20$ & $0.95 \pm 0.03$ & $0.49 \pm 0.16$ & 0.51 & $14.51 \pm 4.31$ & $1.05 \pm 0.49$ & \\
\hline \multirow[t]{11}{*}{1999 Kocaeli, Turkey } & Hotel Sapanca SH-4 [1] & $7.40 \pm 0.11$ & Yes & B & $1.2-2.0$ & 0.50 & $28.10 \pm 5.07$ & $17.31 \pm 2.31$ & $0.37 \pm 0.09$ & $0.96 \pm 0.03$ & $0.41 \pm 0.12$ & 0.70 & $3.25 \pm 1.41$ & $0.45 \pm 0.29$ & $\begin{array}{l}\text { [1] PEER (2000a); } \\
\text { Cetin (personal } \\
\text { communication, } \\
\text { 2002); [2] PEER (2000b); } \\
\text { Sancio et al. (2002, personal } \\
\text { communication, 2002) }\end{array}$ \\
\hline & Soccer Field SF-5 [1] & & Yes & B & $1.2-2.4$ & 1.00 & $30.30 \pm 3.90$ & $22.45 \pm 2.48$ & $0.37 \pm 0.13$ & $0.96 \pm 0.04$ & $0.34 \pm 0.10$ & 0.55 & $2.97 \pm 1.84$ & $1.17 \pm 0.86$ & \\
\hline & Police Station Site [1] & & Yes & B & $1.8-2.8$ & 1.00 & $39.55 \pm 3.38$ & $26.80 \pm 2.48$ & $0.40 \pm 0.10$ & $0.94 \pm 0.05$ & $0.36 \pm 0.10$ & 0.54 & $2.33 \pm 0.47$ & $1.89 \pm 0.55$ & \\
\hline & Yalova Harbor YH-3 [1] & & Yes & B & $3.0-4.5$ & 1.00 & $63.60 \pm 14.93$ & $39.40 \pm 3.12$ & $0.37 \pm 0.13$ & $0.90 \pm 0.07$ & $0.39 \pm 0.11$ & 0.57 & $8.10 \pm 0.66$ & $0.43 \pm 0.07$ & \\
\hline & Adapazari Site B [2] & & Yes & B & $3.3-4.3$ & 3.30 & $60.40 \pm 3.86$ & $55.50 \pm 3.10$ & $0.40 \pm 0.10$ & $0.89 \pm 0.07$ & $0.25 \pm 0.07$ & 0.65 & $5.77 \pm 2.62$ & $0.77 \pm 0.42$ & \\
\hline & Adapazari Site C2 [2] & & Yes & B & $3.3-4.8$ & 0.44 & $73.61 \pm 5.26$ & $38.19 \pm 3.41$ & $0.40 \pm 0.10$ & $0.88 \pm 0.08$ & $0.44 \pm 0.13$ & 0.64 & $3.22 \pm 1.87$ & $1.03 \pm 0.76$ & \\
\hline & Adapazari Site D [2] & & Yes & B & $1.8-2.5$ & 1.50 & $35.28 \pm 2.56$ & $28.90 \pm 2.39$ & $0.40 \pm 0.10$ & $0.95 \pm 0.04$ & $0.30 \pm 0.08$ & 0.75 & $3.54 \pm 1.82$ & $0.58 \pm 0.40$ & \\
\hline & Adapazari Site E [2] & & Yes & B & $1.5-3.0$ & 0.50 & $40.13 \pm 4.85$ & $22.96 \pm 2.75$ & $0.40 \pm 0.10$ & $0.94 \pm 0.05$ & $0.43 \pm 0.13$ & 0.73 & $5.95 \pm 2.76$ & $0.41 \pm 0.27$ & \\
\hline & Adapazari Site F [2] & & Yes & B & $6.8-8.0$ & 0.50 & $42.90 \pm 4.01$ & $67.71 \pm 5.01$ & $0.40 \pm 0.10$ & $0.94 \pm 0.05$ & $0.38 \pm 0.12$ & 0.53 & $4.13 \pm 1.44$ & $0.91 \pm 0.39$ & \\
\hline & Adapazari Site G [2] & & Yes & B & $1.5-2.7$ & 0.45 & $37.50 \pm 3.96$ & $21.31 \pm 2.58$ & $0.40 \pm 0.10$ & $0.95 \pm 0.04$ & $0.43 \pm 0.13$ & 0.84 & $5.03 \pm 1.28$ & $0.32 \pm 0.17$ & \\
\hline & Adapazari Site H [2] & & Yes & B & $2.0-3.0$ & 1.72 & $41.09 \pm 3.43$ & $33.44 \pm 2.56$ & $0.40 \pm 0.10$ & $0.94 \pm 0.05$ & $0.30 \pm 0.18$ & 0.68 & $5.55 \pm 2.03$ & $0.58 \pm 0.31$ & \\
\hline
\end{tabular}


Table 1. (Continued.)

\begin{tabular}{|c|c|c|c|c|c|c|c|c|c|c|c|c|c|c|c|}
\hline Earthquake & Site & $M_{w}$ & Liquefied? & $\begin{array}{l}\text { Data } \\
\text { class }\end{array}$ & $\begin{array}{l}\text { Crit. } \\
\text { depth } \\
\text { range } \\
(\mathrm{m})\end{array}$ & $\begin{array}{c}\text { Depth } \\
\text { to } \\
\text { GWT } \\
\text { (m) }\end{array}$ & $\begin{array}{c}\sigma_{\mathrm{vo}} \\
(\mathrm{kPa})\end{array}$ & $\begin{array}{c}\sigma_{\mathrm{vo}}^{\prime} \\
(\mathrm{kPa})\end{array}$ & $\begin{array}{c}a_{\max } \\
(\mathrm{g})\end{array}$ & $r_{d}$ & CSR & $c$ & $\begin{array}{c}q_{c 1} \\
(\mathrm{MPa})\end{array}$ & $\begin{array}{c}R_{f} \\
(\%)\end{array}$ & Reference \\
\hline & Adapazari Site I [2] & & Yes & B & $3.0-3.5$ & 0.71 & $58.00 \pm 2.46$ & $33.08 \pm 2.69$ & $0.40 \pm 0.10$ & $0.91 \pm 0.06$ & $0.42 \pm 0.11$ & 0.72 & $3.85 \pm 1.04$ & $0.56 \pm 0.32$ & \\
\hline & Adapazari Site J [2] & & Yes & B & $2.5-3.5$ & 0.60 & $44.45 \pm 6.36$ & $30.16 \pm 2.75$ & $0.40 \pm 0.10$ & $0.94 \pm 0.05$ & $0.43 \pm 0.12$ & 0.65 & $3.77 \pm 1.41$ & $0.80 \pm 0.46$ & \\
\hline & Adapazari Site K [2] & & Yes & B & $2.0-3.0$ & 0.80 & $43.85 \pm 3.43$ & $27.17 \pm 2.55$ & $0.40 \pm 0.10$ & $0.94 \pm 0.05$ & $0.39 \pm 0.11$ & 0.62 & $4.19 \pm 1.64$ & $0.91 \pm 0.49$ & \\
\hline & Adapazari Site L [2] & & Yes & B & $2.0-2.8$ & 1.72 & $38.78 \pm 2.75$ & $32.35 \pm 2.46$ & $0.40 \pm 0.10$ & $0.94 \pm 0.05$ & $0.29 \pm 0.08$ & 0.75 & $2.61 \pm 1.24$ & $0.57 \pm 0.36$ & \\
\hline \multirow[t]{16}{*}{1999 Chi-Chi, Taiwan } & Nantou Site C & $7.60 \pm 0.10$ & Yes & B & $2.0-4.5$ & 1.00 & $58.75 \pm 8.12$ & $36.68 \pm 3.65$ & $0.38 \pm 0.08$ & $0.92 \pm 0.06$ & $0.36 \pm 0.10$ & 0.56 & $4.46 \pm 2.07$ & $1.11 \pm 0.62$ & PEER (2000b); Stewart (2001) \\
\hline & WuFeng Site B & & Yes & B & $2.5-5.0$ & 1.12 & $77.39 \pm 8.25$ & $46.68 \pm 3.92$ & $0.60 \pm 0.12$ & $0.85 \pm 0.08$ & $0.59 \pm 0.15$ & 0.55 & $3.22 \pm 1.19$ & $0.96 \pm 0.61$ & \\
\hline & WuFeng Site C & & Yes & B & $2.5-5.5$ & 1.20 & $72.40 \pm 9.74$ & $44.93 \pm 4.19$ & $0.60 \pm 0.12$ & $0.86 \pm 0.08$ & $0.59 \pm 0.16$ & 0.65 & $3.16 \pm 0.73$ & $1.84 \pm 1.33$ & \\
\hline & WuFeng Site A & & Yes & B & $5.5-8.5$ & 0.80 & $130.60 \pm 10.35$ & $69.78 \pm 5.46$ & $0.60 \pm 0.12$ & $0.71 \pm 0.12$ & $0.56 \pm 0.16$ & 0.75 & $0.99 \pm 0.38$ & $2.14 \pm 0.66$ & \\
\hline & WuFeng Site C-10 & & Yes & в & $2.5-7.0$ & 1.00 & $87.25 \pm 14.49$ & $50.46 \pm 5.65$ & $0.60 \pm 0.12$ & $0.82 \pm 0.09$ & $0.60 \pm 0.18$ & 0.58 & $2.52 \pm 1.36$ & $2.18 \pm 2.16$ & \\
\hline & Yuanlini C-19 & & Yes & B & $4.0-5.8$ & 0.57 & $121.79 \pm 6.92$ & $63.62 \pm 4.71$ & $0.25 \pm 0.05$ & $0.82 \pm 0.11$ & $0.25 \pm 0.07$ & 0.67 & $2.78 \pm 0.54$ & $1.08 \pm 0.29$ & \\
\hline & Yuanlin C-2 & & Yes & B & $2.5-4.0$ & 0.56 & $60.07 \pm 5.14$ & $33.68 \pm 3.11$ & $0.25 \pm 0.05$ & $0.93 \pm 0.06$ & $0.27 \pm 0.07$ & 0.75 & $4.95 \pm 1.55$ & $0.49 \pm 0.28$ & \\
\hline & Yuanlin C-22 & & Yes & B & $2.8-4.2$ & 1.13 & $63.11 \pm 4.83$ & $39.86 \pm 3.01$ & $0.25 \pm 0.05$ & $0.92 \pm 0.07$ & $0.24 \pm 0.06$ & 0.70 & $5.17 \pm 0.70$ & $0.46 \pm 0.17$ & \\
\hline & Yuanlin C-24 & & Yes & B & $5.2-7.8$ & 1.20 & $114.20 \pm 7.19$ & $65.15 \pm 4.39$ & $0.25 \pm 0.05$ & $0.83 \pm 0.11$ & $0.24 \pm 0.06$ & 0.75 & $5.33 \pm 1.24$ & $0.60 \pm 0.26$ & \\
\hline & Yuanlin C-25 & & Yes & B & $9.5-12.0$ & 3.52 & $193.69 \pm 9.49$ & $122.76 \pm 6.11$ & $0.25 \pm 0.05$ & $0.67 \pm 0.18$ & $0.17 \pm 0.06$ & 0.61 & $6.83 \pm 0.97$ & $0.80 \pm 0.19$ & \\
\hline & Yuanlin C-32 & & Yes & B & $4.5-7.5$ & 0.74 & $111.78 \pm 10.13$ & $60.18 \pm 5.03$ & $0.25 \pm 0.05$ & $0.84 \pm 0.11$ & $0.25 \pm 0.07$ & 0.70 & $4.83 \pm 1.49$ & $0.62 \pm 0.27$ & \\
\hline & Yuanlin C-4 & & Yes & B & $3.0-6.0$ & 0.66 & $83.52 \pm 9.86$ & $45.85 \pm 4.47$ & $0.25 \pm 0.05$ & $0.89 \pm 0.08$ & $0.26 \pm 0.07$ & 0.55 & $4.60 \pm 1.09$ & $1.30 \pm 1.34$ & \\
\hline & Nantou Site C-8 & & Yes & B & $5.0-9.0$ & 1.00 & $130.00 \pm 13.28$ & $71.14 \pm 6.03$ & $0.38 \pm 0.08$ & $0.77 \pm 0.12$ & $0.35 \pm 0.10$ & 0.55 & $3.31 \pm 0.34$ & $2.08 \pm 0.40$ & \\
\hline & Nantou Site C-7 & & Yes & B & $2.5-4.5$ & 1.00 & $63.50 \pm 6.63$ & $38.98 \pm 3.38$ & $0.38 \pm 0.08$ & $0.91 \pm 0.07$ & $0.37 \pm 0.09$ & 0.76 & $2.31 \pm 0.87$ & $0.57 \pm 0.43$ & \\
\hline & Nantou Sites C-3 \& C-16 & & Yes & $\mathrm{C}$ & $\begin{array}{c}12.0- \\
16.0\end{array}$ & 1.00 & $263.00 \pm 15.19$ & $135.47 \pm 9.53$ & $0.38 \pm 0.08$ & $0.55 \pm 0.20$ & $0.26 \pm 0.11$ & 0.74 & $1.21 \pm 0.23$ & $1.96 \pm 1.13$ & \\
\hline & Yuanlin C-3 & & No & $\mathrm{C}$ & $\begin{array}{c}10.0- \\
13.0\end{array}$ & 1.79 & $218.88 \pm 11.65$ & $123.62 \pm 7.44$ & $0.25 \pm 0.05$ & $0.65 \pm 0.19$ & $0.19 \pm 0.07$ & 0.75 & $6.74 \pm 0.83$ & $0.30 \pm 0.14$ & \\
\hline
\end{tabular}

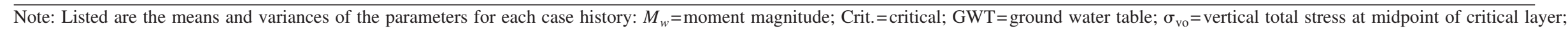

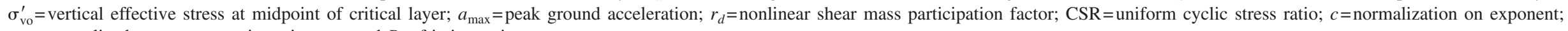
$q_{c 1}=$ normalized average cone tip resistance; and $R_{f}=$ friction ratio.

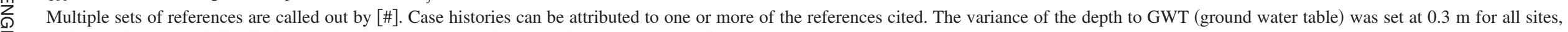
and treated as normal distribution centered on the mean and truncated at the ground surface. 
A full discussion of the application of Bayesian methods to liquefaction triggering analysis can be found in Cetin et al. (2002) and Moss et al. (2003a). In brief, Bayes rule was used as the foundation of the probabilistic analysis; a limit-state function was formulated to define the liquefaction/nonliquefaction threshold; a likelihood function was formulated to describe the observations of liquefaction/nonliquefaction events; Bayesian updating was used to determine the distribution of the unknown model parameters in the limit-state function; and component reliability analysis was used to assess the probability of liquefaction based on the limit-state function with the posterior model parameter distributions. This process can be thought of as a regression-type procedure that has been formulated to incorporate different forms of parameter uncertainty as well as model uncertainty, thereby producing a "best" estimate of the probability of liquefaction triggering.

Bayes rule is derived from simple rules of conditional probability and can be written as (Box and Tiao 1992)

$$
f(\Theta)=C \cdot L(\Theta) \cdot p(\Theta)
$$

where $L(\Theta)=$ likelihood function containing an unknown set of parameters $\Theta ; \quad p(\Theta)=$ prior distribution; $C=\left[\int L(\Theta) \cdot p(\Theta) \cdot d(\Theta)\right]^{-1}$ normalizing constant; and $f(\Theta)$ $=$ posterior distribution.

The likelihood function is proportional to the conditional probability of observing a particular event given a set of parameters. The likelihood function incorporates the objective information that, in this case, is the statistical measurements associated with the observations of liquefaction/nonliquefaction events. The prior distribution can include subjective information known about the distributions of the model parameters. The posterior distribution incorporates both the objective and subjective information into the distributions of the model parameters. The process of performing Bayesian updating involves formulating the likelihood function, selecting a prior, calculating the normalizing constant, and then calculating the posterior statistics.

The prior distribution tends to be a controversial issue with Bayesian methods. Box and Tiao (1992) have shown that, when no prior information is available, the use of a noninformative prior can lead to an unbiased estimate of model parameters. A noninformative prior allows the data, through the likelihood function, to fully dictate the posterior distribution. A noninformative prior has no effect on the shape of the posterior distribution, and is used when no prior information about the parameters is available. In this study a noninformative prior was used for all model parameters.

\section{Limit-State and Model Formulation}

A component reliability problem is formulated in terms of a limitstate function. The limit-state function assumes the value of zero wherever load and resistance values are equal. This defines two regions: one a failure region where the limit-state function is negative and the other a safe region where the limit-state function has a positive value. The probability of failure is the integration of the joint probability density of the load and the resistance over the failure region (Der Kiureghian 1999).

The limit-state can be defined in any manner that corresponds to the observations. Some parameter estimation methods such as system identification and artificial neural networks define the limit-state using a "black box" approach, optimizing a randomly determined mathematical function for the given database. This may give a good fit to a specific database but offers little or no insight into the fundamental phenomena controlling the results. The approach used in this study was to define the limit-state using an understanding of the physics of liquefaction, the knowledge garnered from laboratory experiments, and past deterministic and probabilistic studies.

Using a limit-state that is grounded in an understanding of soil mechanics makes the model fitting a numerical experiment in liquefaction. The limit-state is a generalized mathematical model for separating liquefaction from nonliquefaction cases. By incorporating all pertinent variables and using the database to test the model, an optimum function can be defined that then may give further insight into soil behavior.

The limit-state function $(g)$ used in this study has the form

$$
g=\hat{g}+\varepsilon
$$

where $\quad \hat{g}=q_{c, 1} \cdot\left(1+\theta_{1} \cdot R_{f}\right)+\left(\theta_{2} \cdot R_{f}\right)+c \cdot\left(1+\theta_{3} \cdot R_{f}\right)-\theta_{4} \cdot \ln (\mathrm{CSR})$ $-\theta_{5} \cdot \ln \left(M_{w}\right)-\theta_{6} \cdot \ln \left(\sigma_{v}^{\prime}\right)-\theta_{7} ;$ and $\mathrm{CSR}=$ simplified cyclic stress ratio; $M_{w}=$ moment magnitude; $\sigma_{v}^{\prime}=$ effective stress; $q_{c, 1}=$ normalized CPT tip resistance; $R_{f}=$ friction ratio; $c=\mathrm{CPT}$ normalization exponent; $\theta$ 's $=$ model parameters; and the $\varepsilon=$ model error term. The limit-state is the threshold between liquefaction $(g \leqslant 0)$ and nonliquefaction $(g>0)$. The model error term $\varepsilon$ is treated as a standard normal variate with zero mean and an unknown standard deviation. Through Bayesian updating, we estimate the model parameters $\theta_{1}-\theta_{7}$ and the standard deviation of $\varepsilon$.

This particular functional form of the limit-state was chosen from a suite of models tested against the database, because it minimized the standard deviation of the model error, minimized cross correlation of the independent variables, and provided mathematical flexibility so as to not impose arbitrary constraints on the model fit. The various models tested were found to have relatively consistent mean locations; and it was the magnitude of the model error and cross-correlation terms that separated the optimum model from the other models.

\section{Bayesian Updating}

The process of model parameter estimation, or testing the limitstate function against the data, is accomplished through Bayesian updating. According to Bayes rule [i.e., Eq. (12)], to solve for the posterior distribution of the model parameters and model error term we must define the likelihood function, determine a prior distribution, and solve for the normalizing constant.

The likelihood function of seismic soil liquefaction initiation is the product of the probabilities of observing $k$ liquefied sites and $n-k$ nonliquefied sites. Thus

$$
L(X, \Theta, \varepsilon) \propto P\left\{\bigcap_{i=1}^{k}\left[\hat{g}\left(X_{i}, \Theta\right)+\varepsilon_{i} \leqslant 0\right] \bigcap_{i=k+1}^{n}\left[\hat{g}\left(X_{i}, \Theta\right)+\varepsilon_{i}>0\right]\right\}
$$

where $X_{i}=$ observable or measurable variables at the $i$ th site; $\Theta=\left(\theta_{1}, \ldots, \theta_{7}\right)=$ set of unknown model parameters, and $\varepsilon_{i}=$ realization of the model error term at the $i$ th site. With $\varepsilon$ a normal random variable with zero mean and standard deviation $\sigma_{\varepsilon}$, the likelihood function becomes

$$
L\left(X, \Theta, \sigma_{\varepsilon}\right) \propto \prod_{i=1}^{k} \Phi\left[-\frac{\hat{g}\left(X_{i}, \Theta\right)}{\sigma_{\varepsilon}}\right] \cdot \prod_{i=k+1}^{n} \Phi\left[\frac{\hat{g}\left(X_{i}, \Theta\right)}{\sigma_{\varepsilon}}\right]
$$

where $\Phi=$ standard normal cumulative distribution function. 
A noninformative prior has been employed in this study, which allows the likelihood function to dominate the posterior distribution. As mentioned earlier, a noninformative prior distribution, by definition, will have as little effect on the posterior distribution as possible. In the present case, the noninformative prior has the form $p\left(\Phi, \sigma_{\varepsilon}\right) \sim 1 / \sigma_{\varepsilon}$ (Box and Tiao 1992).

Importance sampling was used to calculate the normalizing constant integral. This is a simulation technique that efficiently approximates the complex integral numerically (Gardoni et al. 2002).

\section{Sampling Bias}

A bias exists in the number of liquefied versus nonliquefied data points because liquefaction sites are disproportionately selected for postearthquake investigations. This bias can impact the results of statistical analysis, producing a skewed prediction. Cetin et al. (2002) explored this bias and presented a method to account for what is called choice-based sampling bias as applied to the problem of liquefaction triggering.

The approach was based on Bayesian updating optimization, expert consensus, and sensitivity studies. The likelihood function [Eq. (15)] is modified for the data imbalance using a weighting factor $(w)$

$$
\begin{aligned}
L\left(X, \Theta, \sigma_{\varepsilon}\right) \propto & \prod_{i=1}^{k} \Phi\left[-\frac{\hat{g}\left(X_{i}, \Theta\right)}{\sigma_{\varepsilon}}\right]^{w_{\text {liquefied }}} \\
& \times \prod_{i=k+1}^{n} \Phi\left[\frac{\hat{g}\left(X_{i}, \Theta\right)}{\sigma_{\varepsilon}}\right]^{w_{\text {nonliquefied }}}
\end{aligned}
$$

The weighting factor used in this study was $w_{\text {nonliquefied }} / w_{\text {liquefied }}=1.5$, based on Cetin et al. (2002) and consensus of the expert panel that reviewed the CPT database.

\section{Reliability Analysis}

The probability of liquefaction was calculated from the posterior distribution determined through Bayesian updating. It should be noted that the probability associated with Bayesian methods is considered an expression of degree of belief, whereas in classical methods the probability is considered a measure of relative frequency. The probability of liquefaction can be estimated by a summation of the probabilities of all possible combinations of parameters that will define liquefaction. For any given set of variables, $X$, this requires integration over the liquefaction domain $(g \leqslant 0)$. The result is

$$
P[\hat{g}(X, \Theta)+\varepsilon \leqslant 0]=\int_{\hat{g}(X, \Theta)+\varepsilon \leqslant 0} \varphi\left(\varepsilon \mid \sigma_{\varepsilon}\right) \cdot f\left(\Theta, \sigma_{\varepsilon}\right) \cdot d \varepsilon \cdot d \Theta \cdot d \sigma_{\varepsilon}
$$

where $\varphi=$ standard standard normal probability density function.

The solution of Eq. (17) requires multifold integration. Good approximations of the resultant probabilities were achieved using a mean value first order second moment (MVFOSM) approach, because the failure surface was well behaved (i.e., not highly nonlinear). These results were validated using first order reliability method (FORM), second order reliability method (SORM), and Monte Carlo simulations as coded in the program CALREL (Liu et al. 1989).

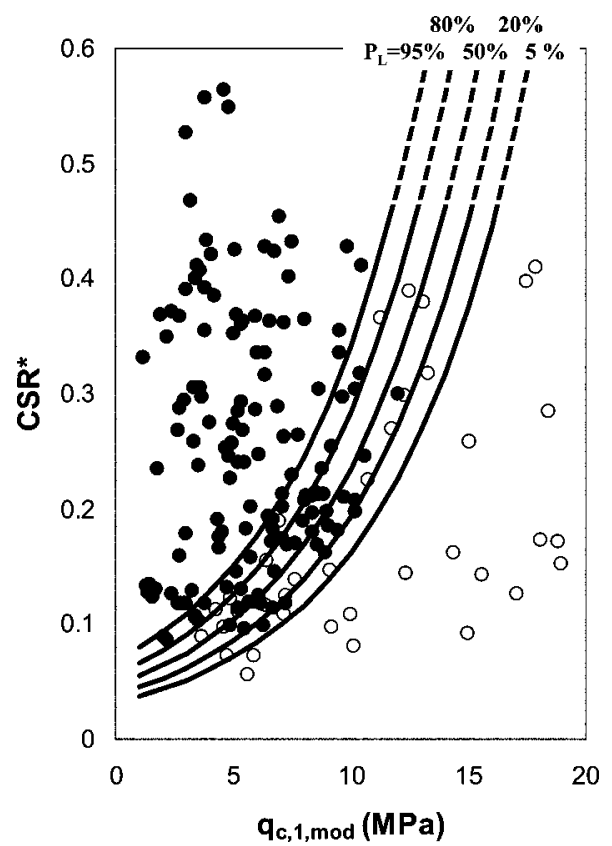

Fig. 6. Contours of 5, 20, 50, 80, and $95 \%$ probability of triggering of liquefaction as a function of duration-corrected equivalent uniform cyclic stress ratio $\left(\mathrm{CSR}^{*}\right)$ and "fines" adjusted CPT tip resistance $\left(q_{c, 1, \mathrm{mod}}\right)$ for $M_{w}=7.5, \sigma_{v}^{\prime}=1 \mathrm{~atm}$. Closed circles are liquefied and open circles are nonliquefied field case histories.

\section{CPT-Based Liquefaction Triggering Correlation}

Fig. 6 presents one view of the new correlation, in this case a plot of contours of probability of liquefaction (for $P_{L}=5,20,50,80$, and $95 \%)$ as a function of the equivalent uniform cyclic stress ratio $\left(\mathrm{CSR}^{*}\right)$ and the modified normalized $\mathrm{CPT}$ tip resistance $\left(q_{c, 1, \text { mod }}\right)$. In this figure, the equivalent uniform CSR has been corrected for duration effects based on the magnitude correlated $\mathrm{DWF}_{M}$. The solid dots represent the centroids of probability distributions of the individual case histories for which liquefaction was triggered and open circles similarly represent nonliquefaction cases. These distributions quantify each individual field case history. The horizontal axis of Fig. 6 represents a modification of normalized CPT tip resistance $\left(q_{c, 1}\right)$ for the frictional effects of apparent "fines" and character. To account for the systematic suppression of liquefiability with increased friction ratio, values of $q_{c, 1}$ are adjusted as follows:

$$
q_{c, 1, \bmod }=q_{c, 1}+\Delta q_{c}
$$

where $\quad \Delta q_{c}=x_{1} \cdot \ln (\mathrm{CSR})+x_{2} ; \quad x_{1}=0.38 \cdot\left(R_{f}\right)-0.19 ; \quad$ and $x_{2}=1.46 \cdot\left(R_{f}\right)-0.73$. In this equation $q_{c, 1}$ is in $\mathrm{MPa}, R_{f}$ in percent, and the bounds of $\Delta q_{c}$ are from $R_{f}=0.5-5.0$, where $\Delta q_{c}=0$ when $R_{f} \leqslant 0.5, \Delta q_{c}$ reaches its maximum at $R_{f}=5.0$, and no data exist for $R_{f}>5.0$. This "fines" adjustment was regressed from the liquefaction database and represents the change in liquefiability correlated to the change in friction ratio, as a function of CSR.

Fig. 7 shows a comparison of the proposed probability curves with the "clean" sand curves from Shibata and Teparaska (1988), Robertson and Wride (1998), and Idriss and Boulanger (2004), as well as the $P_{L}=50 \%$ curves from Toprak et al. (1999) and Juang et al. (2003). The comparison shows general agreement between the proposed probabilistic curves and the previous deterministic and probabilistic correlations. Of particular interest is the comparison to the "clean" sand $\left(K_{C}=1.0\right)$ curve by Robertson and 


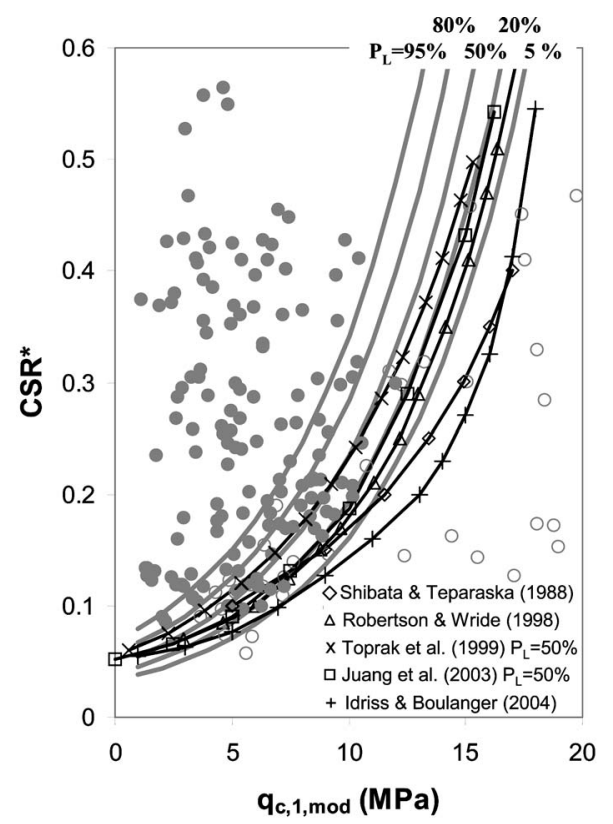

Fig. 7. Comparison of recommended curves with previous deterministic and probabilistic "clean" sand curves

Wride, which forms the basis for current practice. That triggering curve is generally compatible with a $P_{L}=15 \%$ curve, which was selected as the probability of liquefaction to represent a deterministic boundary.

Fig. 8 shows the multiplicative "fines" adjustment factors recommended by Robertson and Wride (1998), denoted $K_{C}$, along with the additive adjustment factors from this study. The multiplicative adjustment corrects the depth-normalized CPT tip resistance as follows:

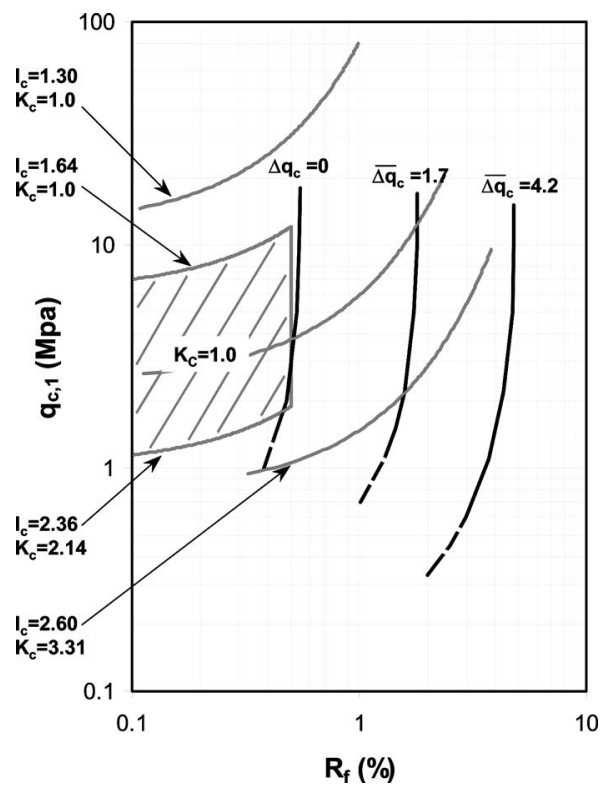

Fig. 8. Comparison of recommended $\left(\Delta q_{c}\right)$ "fines" adjustment versus previous $\left(K_{c}\right)$ recommendations, adapted from Robertson and Wride (1998)

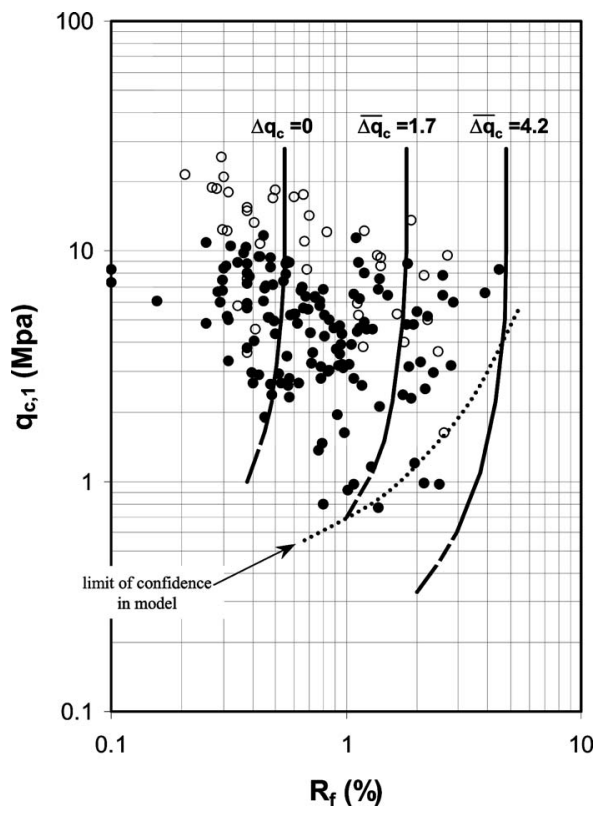

Fig. 9. Recommended "fines" adjustment shown with liquefied (closed circles) and nonliquefied (open circles) field case histories. Dotted line indicates limit of confidence in model.

$$
q_{c, 1, \bmod }=q_{c, 1} \cdot K_{C}
$$

where $K_{C}=$ function of both tip resistance and friction ratio. The factor $K_{C}$ is taken as 1.0 (a null adjustment of $q_{c, 1}$ ) in the crosshatched region of Fig. 8.

The proposed "fines" adjustment, denoted $\Delta q_{c}$, is also a null adjustment in this shaded zone, but more smoothly transitions to finite adjustments as $q_{c, 1}$, decreases or $R_{f}$ increases. The new contours also provide for smaller adjustments of $q_{c, 1}$ than the factors of Robertson and Wride. In fitting an equation to the observed "fines" adjustment surface, it was found that an additive equation captured the behavior better than a multiplicative equation. We suggest that the proposed "fines" adjustment is founded on how the increase in friction ratio affects the liquefiability of a material, whereas the Robertson and Wride "fines" adjustment is founded on soil classification that relies on the correlation of CPT measurements to soil behavior type, and not necessarily the liquefiability of a material.

Fig. 9 shows the $\Delta q_{c}$ adjustment curves with respect to the liquefaction database. The dashed line at the bottom indicates the limit of confidence in the model. This is a recommended limit for materials that may have a texture that is conducive to liquefaction based on the existing database. However, clayey soils may also lie above the line requiring soil samples when the soil texture is in doubt.

Fig. 10 presents an alternate, deterministic, view of the new correlation. In this case, contours of $P_{L}=15 \%$ are shown for three different values of mean $\Delta q_{c}$ spanning the full available range of $\Delta q_{c}$. These curves also approximately represent contours of equal friction ratio $\left(R_{f}\right)$.

Fig. 11 provides a comparison between the new proposed CPT-based deterministic curves, and previous deterministic curves proposed by Robertson and Wride (1998) and Suzuki et al. (1995). The "fines" adjustments of Suzuki et al. suggest a trend similar to those from this study, but are biased to the right of the proposed $P_{L}=15 \%$ curves. The adjustments from this study are much smaller than those proposed by Robertson and Wride. Idriss 


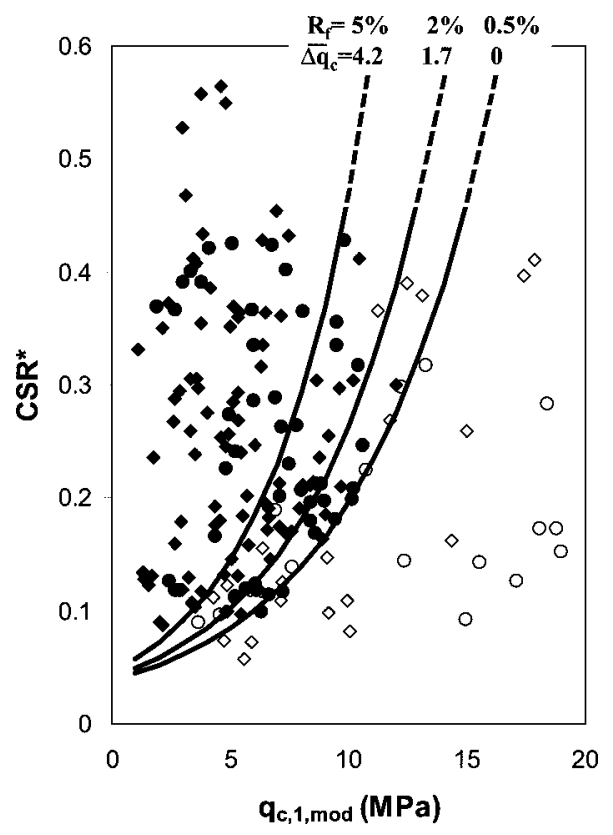

Fig. 10. Recommended deterministic triggering correlation. This figure presents "fines" adjusted triggering curves, all shown for $P_{L}$ $=15 \%$. Circles (closed and open) indicate "clean" sands $\left(R_{f}\right.$ $\leqslant 0.5 \%$ ) and diamonds (closed and open) indicate soils of higher apparent "fines" content $\left(R_{f}>0.5 \%\right)$, all for $M_{w}=7.5, \sigma_{v}^{\prime}=1 \mathrm{~atm}$.

and Boulanger (2004) present a comparison of the Robertson and Wride curves with the Moss et al. (2003b) case histories with appreciable apparent fines content, which demonstrates the reason for the difference in fines adjustments.

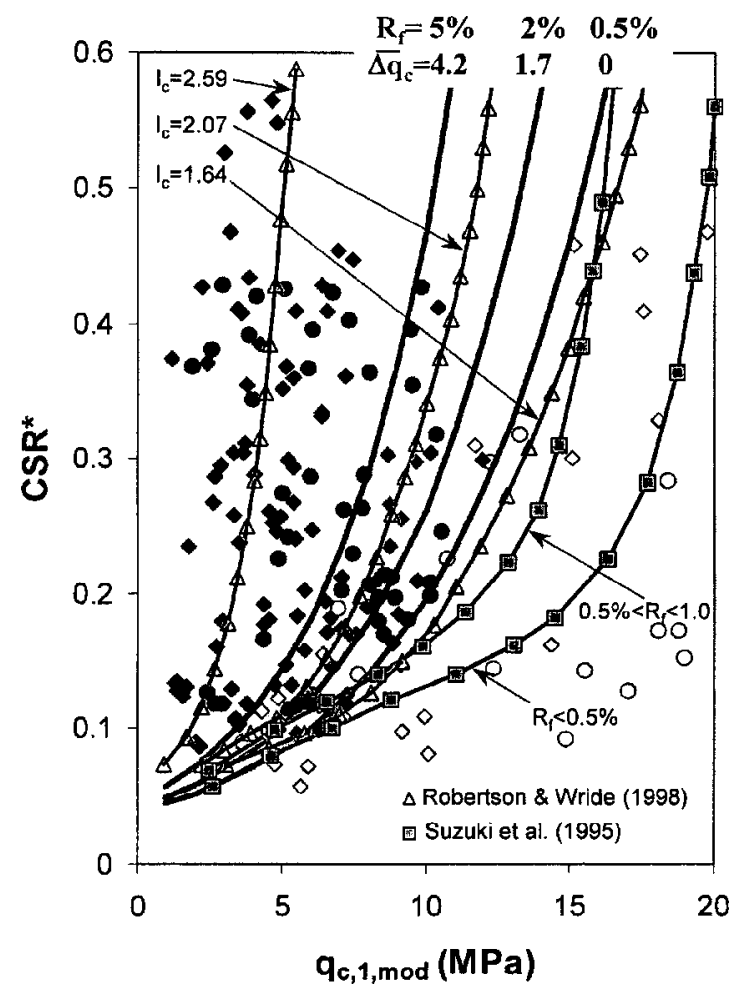

Fig. 11. Comparison of "fines" adjusted $\left(P_{L}=15 \%\right)$ triggering curves with previous similar curves

For exact parameter estimation (assuming mean values), the following equation can be used to calculate the probability of liquefaction:

$$
P_{L}=\Phi\left\{-\frac{\left[q_{c, 1}^{1.045}+q_{c, 1}\left(0.110 \cdot R_{f}\right)+\left(0.001 \cdot R_{f}\right)+c\left(1+0.850 \cdot R_{f}\right)-7.177 \cdot \ln (\mathrm{CSR})-0.848 \cdot \ln \left(M_{w}\right)-0.002 \cdot \ln \left(\sigma_{v}^{\prime}\right)-20.923\right]}{1.632}\right\}
$$

where $q_{c, 1}=$ normalized tip resistance $(\mathrm{MPa}) ; R_{f}=$ frictionratio (percent); $c=$ normalization exponent; CSR=equivalent uniform cyclic stress ratio; $\sigma_{v}=$ effective overburden stress (kilopascal); and $\Phi\left(P_{L}\right)=$ cumulative normal distribution. The cyclic resistance ratio for a given probability of liquefaction can be calculated from

$$
\mathrm{CRR}=\exp \left\{\frac{\left[q_{c, 1}^{1.045}+q_{c, 1}\left(0.110 \cdot R_{f}\right)+\left(0.001 \cdot R_{f}\right)+c\left(1+0.850 \cdot R_{f}\right)-0.848 \cdot \ln \left(M_{w}\right)-0.002 \cdot \ln \left(\sigma_{v}^{\prime}\right)-20.923+1.632 \cdot \Phi^{-1}\left(P_{L}\right)\right]}{7.177}\right\}
$$

where $\Phi^{-1}\left(P_{L}\right)=$ inverse cumulative normal distribution function. [In an Excel spreadsheet the cumulative normal distribution, $\Phi\left(P_{L}\right)$, can be calculated using "NORMDIST $\left(P_{L}, 0,1\right.$, TRUE)" and the inverse cumulative normal distribution function, $\Phi^{-1}\left(P_{L}\right)$, can be calculated using "NORMINV $\left(P_{L}, 0,1\right)$."] Note that Eq. (21) produces results for the input magnitude $\left(\mathrm{CRR}_{M_{w}=\text { ? }}\right)$, whereas Figs. 6 and 10 give results for a mean magnitude of 7.5 (CSR ${ }^{*}$ ). The liquefaction triggering results presented in this paper can be used in forward analyses either in a probabilistic or deterministic manner.

Normalized tip resistance $\left(q_{c, 1}\right)$ and the normalization exponent $(c)$ were incorporated into the limit-state function, which we believe provides a comprehensive assessment of the influence of effective overburden on CPT measurements and liquefaction for free field conditions. The statistical results showed that the effective overburden stress became an unimportant, or noncontributing, variable in the analysis when the CPT measurements were properly normalized. Therefore, we suggest that using the recommended iterative normalization scheme obviates the need for subsequent $K_{\sigma}$ corrections commonly applied to liquefaction analysis, and these corrections were not applied in this study.

The upper portion of the curves, with CSR greater than $\sim 0.4$, are not adequately constrained by data in this or previous studies. This high CSR region of liquefaction correlations continues to be 
controversial. Some researchers believe that the curves should approach a vertical asymptote and that no further liquefaction is possible after a certain limiting resistance. However, even very dense granular materials can contract during cyclic shearing, leading to "initial liquefaction" as defined by Seed (1979), although the strain potential associated with this liquefaction would be extremely limited. The curves developed in this study have a slight "lean" in the high CSR range that is consistent with the limited data and reflects the authors' judgment on this issue.

\section{Summary}

In this paper we present a new correlation for CPT-based assessments of seismically induced soil liquefaction hazard. The new correlation employs a larger database of high quality field performance case histories than was available to previous researchers. In processing the case history data, we have screened the data to remove cohesive soil sites, sites with significant static shear stresses, and nonliquefaction sites that may in fact have liquefied at depth without surface manifestation. We have utilized new normalization procedures for CPT resistance as presented in Moss et al. (2006).

Overall, the new correlation is in general agreement with previous efforts with regard to "clean" sands. It is principally when evaluating silts and sand with appreciable fines that the new correlation differs significantly from earlier and widely used CPTbased correlations. The new curves reflect a much smaller modification in CPT tip resistance with an increase in apparent "fines" content than the earlier relationships. We believe that the proposed curves accurately capture the entire range of potentially liquefiable materials that the CPT can be used to measure.

\section{Acknowledgments}

Financial support was provided by the California Department of Transportation (CalTrans), the California Energy Commission (CEC), and Pacific Gas and Electric Company (PG\&E) through the Pacific Earthquake Engineering Research (PEER) Center's Lifelines Program, Task 3D02. This support is greatly appreciated. The writers would like to thank Daniel Chu's contribution of the Taiwan data and review of the processed Taiwan case histories. Thanks also go to Professor Emeritus T. Leslie Youd for reviewing a portion of the database. The comments and suggestions by Professor Emeritus I. M. Idriss, Professor Emeritus T. Leslie Youd, and Professor Geoffrey Martin during the PEER quarterly meetings were very useful in guiding this work. Thanks also go to Professor K. Tokimatsu and Dr. Y. Suzuki for providing useful data from the Kobe earthquake, and the anonymous reviewers who provided comments that helped them improve this paper.

\section{Notation}

The following symbols are used in this paper:

$a_{\max }=$ maximum horizontal ground acceleration;

$\mathrm{CSR}=$ cyclic stress ratio;

$\mathrm{CSR}^{*}=$ duration corrected cyclic stress ratio;

$C=$ Bayes normalizing constant;

$C_{q}=$ tip normalization factor;

$C_{s}=$ sleeve normalization factor;

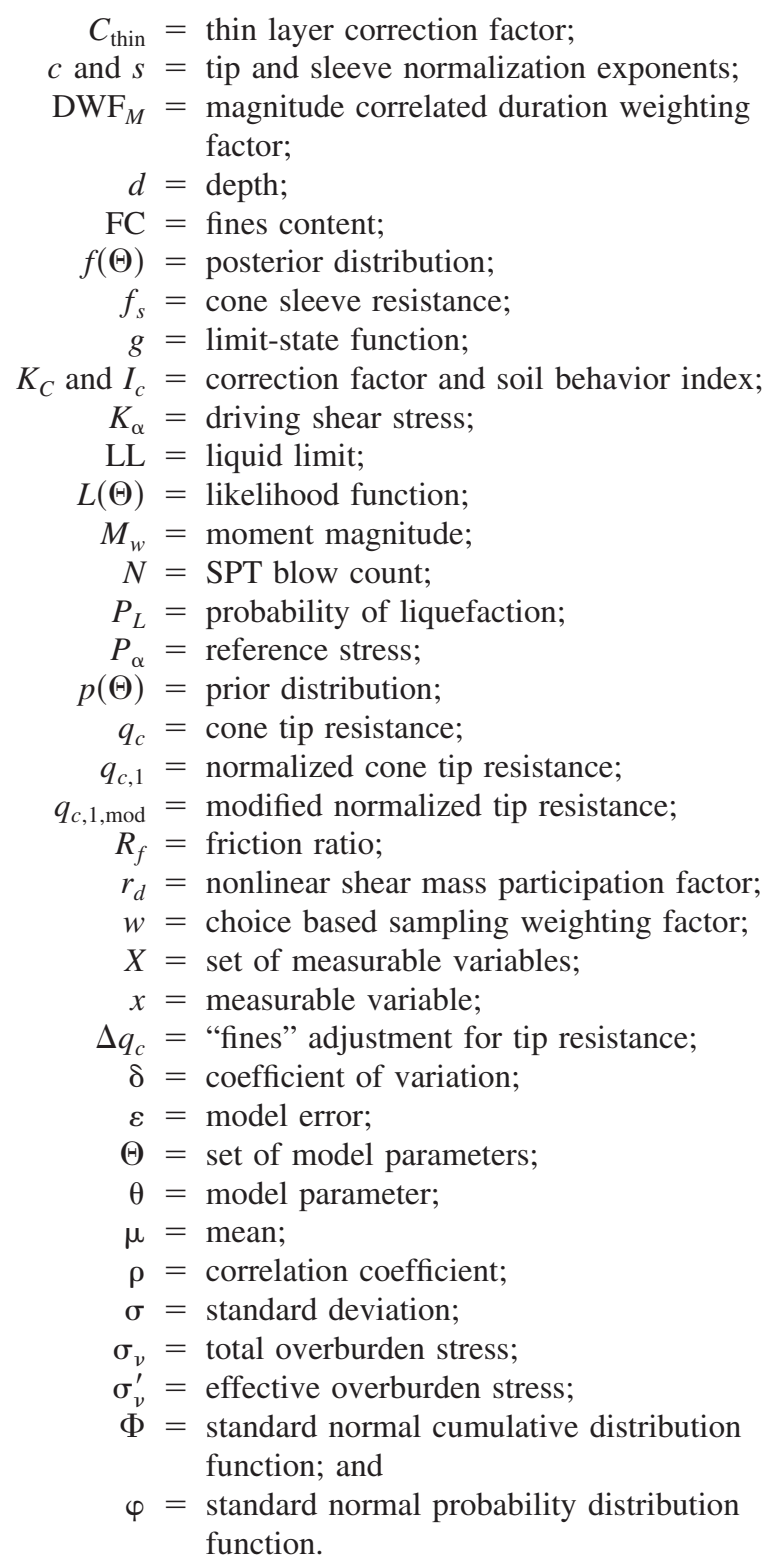

\section{References}

Albel-Haq, A., and Hryciw, R. D. (1998). "Ground settlement in Simi Valley following the Northridge Earthquake." J. Geotech. Geoenviron. Eng., 124(1), 80-89.

Anderson, J. G., and Simons, R. S. (1982). "The Mexicali Valley Earthquake of 9 June 1980." Earthquake Engineering Research Institute Newsletter, 16(3), 73-105.

Andrews, D. C. A., and Martin, G. R. (2000). "Criteria for liquefaction of silty soils." Proc., 12th World Conf. Earthquake Engineering, Auckland, New Zealand, Paper 0312.

Andrianopoulos, K. I., Bouckovalas, G. D., and Papadimitriou, A. G. (2001). "A critical state evaluation of fines effect on liquefaction potential." Proc., 4th Int. Conf. on Recent Advances in Geotechnical Earthquake Engineering and Soil Dynamics, Univ. Missouri-Rolla, Mo., Paper No. 4.06.

Andrus, R. D., Stokoe, K. H., II, and Roesset, J. M. (1991). "Liquefaction of gravelly soil at Pence Ranch during the 1983 Borah Peak, Idaho Earthquake." Soil dynamics and earthquake engineering, Vol. 5, Computational Mechanics Publications and Elsevier Applied Science, London.

Arulanandan, K., Douglas, B. J., Qu, Y. Z., Junfei, X., Chengchun, W., 
and Qizhi, H. (1982). "Evaluation of earthquake induced liquefaction in Tientsin during the Tangshan Earthquake P. R. C." Proc., United States-People's Republic of China Bilateral Workshop on Earthquake Engineering, E-3-1-E-3-42.

Arulanandan, K., Yogachandran, C., Meegoda, N. J., Ying, L., and Zhauji, S. (1986). "Comparison of the SPT, CPT, SV and electrical methods of evaluating earthquake induced liquefaction susceptibility in Ying Kou City during the Haicheng Earthquake. Use of in situ tests in geotechnical engineering." Geotech. Spec. Publ., 6, 389-415.

Bennett, M. J., McLaughlin, P. V., Sarmiento, J. S., and Youd, T. L. (1984). "Geotechnical investigation of liquefaction sites, Imperial Valley, California." Open File Rep. 84-252, Dept. of the Interior, U.S. Geological Survey, Menlo Park, Calif.

Bennett, M. J., Ponti, D. J., Tinsley, J. C., Holzer, T. L., and Conaway, C. H. (1998). "Subsurface geotechnical investigations near sites of ground deformation caused by the January 17, 1994, Northridge, California, Earthquake." U. S. G. S. Open-File Rep. No. 98-373, Menlo Park, Calif.

Bennett, M. J., and Tinsley, J. C. I. (1995). "Geotechnical data from surface and subsurface samples outside of and within liquefactionrelated ground failures caused by the October 17, 1989, Loma Prieta Earthquake, Santa Cruz and Monterey Counties, California." OpenFile Rep. 95-663, U.S. Dept. of the Interior, U.S. Geological Survey, Menlo Park, Calif.

Bierschwale, J. G., and Stokoe, K. H., II. (1984). "Analytical evaluation of liquefaction potential of sands subjected to the 1981 Westmoreland Earthquake." Geotechnical Engineering Rep. No. GR 84-15, Univ. of Texas, Austin, Tex.

Boulanger, R. W. (2003). "High overburden stress effects in liquefaction analyses." J. Geotech. Geoenviron. Eng., 129(12), 1071-1082.

Boulanger, R. W., and Idriss, I. M. (2004). "Evaluating the potential for liquefaction or cyclic failure of silts and clays." Rep. UCD/CGM-04/ 01, Dept. of Civil and Environmental Engineering, College of Engineering, Univ. of California at Davis, Davis, Calif.

Boulanger, R. W., Idriss, I. M., and Mejia, L. H. (1995). "Investigation and evaluation of liquefaction related ground displacements at Moss Landing during the 1989 Loma Prieta Earthquake." Rep. UCD/CGM95/02, Center for Geotechnical Modeling, Dept. of Civil and Environmental Engineering, Univ. of California Davis, Davis, Calif.

Boulanger, R. W., Mejia, L. H., and Idriss, I. M. (1997). "Liquefaction at Moss Landing during Loma Prieta Earthquake.” J. Geotech. Geoenviron. Eng., 123(5), 453-467.

Box, G. E. P., and Tao, G. C. (1992). Bayesian inference in statistical analysis, Wiley, New York.

Bray, J. D., Sancio, R. B., Riemer, M. F., and Durgunoglu, T. (2004). "Liquefaction susceptibility of fine-grained soils." Proc., 11th Int. Conf. Soil Dynamics and Earthquake Engineering and 3rd Int. Conf. Earthquake Geotechnical Engineering, Berkeley, Calif.

Cao, L. F., Teh, C. I., and Chang, M. F. (2001). "Undrained cavity expansion in modified cam clay. I: Theoretical analysis." Geotechnique, 51(4), 323-334.

Cetin, K. O. (2000). "Reliability-based assessment of seismic soil liquefaction initiation hazard." Ph.D. dissertation, Univ. of California, Berkeley, Calif.

Cetin, K. O., Der Kiureghian, A., and Seed, R. B. (2002). "Probabilistic models for the initiation of seismic soil liquefaction." Struct. Safety, 24, 67-82.

Cetin, K. O., Seed, R. B., Der Kiureghian, A., Tokimatsu, K., Harder, L. F., Jr., Kayen, R. E., and Moss, R. E. S. (2004). "Standard penetration test-based probabilistic and deterministic assessment of seismic soil liquefaction potential." J. Geotech. Geoenviron. Eng., 130(12), 1314-1340.

Chameau, J. L., Clough, G. W., Reyna, F. A. M., and Frost, J. D. (1991). "Liquefaction response of San Francisco Bayshore fills." Bull. Seismol. Soc. Am., 81(5), 1998-2018.

Christensen, S. A. (1995). "Liquefaction of cohesionless soils in the March, 2, 1987 Edgecumbe Earthquake, Bay of Plenty, New Zealand, and other earthquakes." Master's of Engineering thesis, Univ. of Canterbury, Christchurch, New Zealand.

Chu, D. B., Stewart, J. P., Lee, S., Tsai, J. S., Lin, P. S., Chu, B. L., Seed, R. B., Hsu, S. C., Yu, M. S., and Wang, M. C. H. (2004). "Documentation of soil conditions at liquefaction and non-liquefaction sites from 1999 Chi-Chi (Taiwan) earthquake." Soil Dyn. Earthquake Eng., 24(9-10), 647-657.

De Alba, P., Youd, T. L., Shakal, A. F., Benoit, J., Pass, D. G., and Carter, J. J. (1998). "Deep instrumentation array at the Treasure Island Naval Station." U.S. Geol. Surv. Prof. Pap..

Der Kiureghian, A. (1999). "A Bayesian framework for fragility assessment.” Proc., ICASP8 Conf., Sidney, Australia.

Diaz-Rodriquez, J. A. (1983). "Investigation del comportamiento seismico de suelos granulares finos.” Doctoral thesis, Univ. Nacional Autonoma Mexico, Mexico.

Diaz-Rodriquez, J. A. (1984). "Liquefaction in the Mexicali Valley during the earthquake of June 9, 1980." Proc., 8th World Conf. on Earthquake Engineering, EERI, San Francisco, 223-230.

Dowrick, D. J., and Sritharan, S. (1993). "Peak ground accelerations recorded in the 1968 Inangahua Earthquake and some attenuation implications." New Zealand Nat. Soc. Earthquake Eng. Bull, 26(3), 349-355.

Earth Technology Corporation. (1985). "In situ testing II, Peoples Republic of China." No. 84-141-13.

Farrar, J. A. (1990). "Study of in situ testing for evaluation of liquefaction resistance." Bureau of Reclamation, U.S. Dept of the Interior, R-90-06, Denver.

Franklin, A. G. (1986). "Use of the piezocone for evaluating soil liquefaction potential." Proc., 8th European Conf. on Earthquake Engineering, Lisbon, Portugal, 33-40.

Frost, J. D., Reyna, F. A., Chameau, J. L., and Karanikolas, P. (1993). "Performance of fill soils during the Loma Prieta Earthquake," Proc., 3rd Int. Conf. on Case Histories in Geotechnical Engineering, Vol. 1, St. Louis, 541-546.

Gardoni, P., Der Kiureghian, A., and Mosalam, K. M. (2002). "Probabilistic capacity models and fragility estimates for reinforced concrete columns based on experimental observations." J. Eng. Mech., 128(10), 1024-1038.

Guo, T., and Prakash, S. (1999). "Liquefaction of silts and silt-clay mixtures.” J. Geotech. Geoenviron. Eng., 125(8), 706-710.

Holzer, T. L., Bennett, M. J., Ponti, D. J., and Tinsley, J. C. (1999) "Liquefaction and soil failure during 1994 Northridge Earthquake." $J$. Geotech. Geoenviron. Eng., 125(6), 438-452.

Holzer, T. L., Tinsley, J. C. I., Bennett, M. J., and Mueller, C. S. (1994). "Observed and predicted ground deformation-Miller Farm Lateral Spread, Watsonville, California." Proc., 5th U.S.-Japan Workshop on Earthquake Resistant Design of Lifeline Facilities and Countermeasures for Soil Liquefaction, Technical Rep. NCEER-94-0026 79-99.

Idriss, I. M. (1999). "An update to the Seed-Idriss simplified procedure for evaluating liquefaction potential." Presentation Notes, Proc. Workshop, New Approaches to Liquefaction Analysis, Transportation Research Board, Washington, D.C.

Idriss, I. M., and Boulanger, R. W. (2004). "Semi-empirical procedures for evaluating liquefaction potential during earthquakes." Proc., 11th Int. Conf. Soil Dynamics and Earthquake Engineering and 3rd Int. Conf. Earthquake Geotechnical Engineering, Berkeley, Calif., 32-56.

Inagaki, H., Iai, S., Sugano, T., Yamazaki, H., and Inatomi, T. (1996). "Performance of Caisson type quay walls at Kobe Port." Soils Found., 119-136.

Ishihara, K. (1985). "Stability of natural deposits during earthquakes." Proc., 11th Int. Conf. of Soil Mechanics and Foundation Engineering, Vol. 1, 321-376.

Ishihara, K., and Koga, Y. (1981). "Case studies of liquefaction in the 1964 Niigata Earthquake.” Soils Found., 21(3).

Ishihara, K., and Perlea, V. (1984). "Liquefaction-associated ground damage during the Vrancea Earthquake of March, 4, 1977." Soils Found., 24(1), 90-112.

Jamiolkowski, M., Baldi, G., Bellotti, R., Ghionna, V., and Pasqualini, E. 
(1985). "Penetration resistance and liquefaction of sands." Proc., 11th Int. Conf. on Soil Mechanics and Foundation Engineering, San Francisco, 1891-1896.

Juang, C. H., Yuan, H., Lee, D. H., and Lin, P. S. (2003). "Simplified cone penetration test-based method for evaluating liquefaction resistance of soils." J. Geotech. Geoenviron. Eng., 129(1), 66-80.

Kayen, R. E., Mitchell, J. K., Seed, R. B., and Nishio, S. (1998). "Soil liquefaction in the East Bay during the earthquake." U.S. Geol. Surv. Prof. Pap., 1551-B.

Kurup, P. U., Voyiadjis, G. Z., and Tumay, M. T. (1994). "Calibration chamber studies of piezocone test in cohesive soils." J. Geotech. Eng., 120(1), 81-107.

Ladanyi, B., and Johnston, G. H. (1974). "Behavior of circular footings and plate anchors embedded in permafrost." Can. Geotech. J., 11, 531-553.

Liao, S. S. C., and Whitman, R. V. (1986). "Overburden correction factors for SPT in sand." J. Geotech. Eng., 112(3), 373-377.

Liu, A. H., Stewart, J. P., Abrahamson, N. A., and Moriwaki, Y. (2001). "Equivalent number of uniform stress cycles for soil liquefaction analysis.” J. Geotech. Geoenviron. Eng., 127(12), 1017-1026.

Liu, P., Lin, H., and Der Kiureghian, A. (1989). "CALREL user manual." Structural Engineering Mechanics and Materials Rep. No. UCB/ SEMM-89/18, Univ. of California Berkeley, Berkeley, Calif.

Meyerhoff, G. G. (1976). "Bearing capacity and settlement of pile foundations." J. Geotech. Eng. Div., Am. Soc. Civ. Eng., 102(3), 197-228.

Mitchell, J. K., Lodge, A. L., Coutinho, R. Q., Kayen, R. E., Seed, R. B., Nishio, S., and Stokoe, K. H., II. (1994). "In situ test results from four Loma Prieta Earthquake liquefaction sites: SPT, CPT, DMT, and shear wave velocity." Rep. UCB/EERC-94/04, Earthquake Engineering Research Center, College of Engineering, Univ. of California Berkeley, Berkeley, Calif.

Mitchell, J. K., and Tseng, D. J. (1990). "Assessment of liquefaction potential by cone penetration resistance." Proc., H. Bolton Seed Memorial Symp. Duncan, J. M. BiTech, Vancouver, Canada, 335-350.

Moss, R. E. S. (2003). "CPT-based probabilistic assessment of seismic soil liquefaction initiation." Doctor of Philosophy dissertation, Univ. of California, Berkeley, Calif. 〈http://www.fugrowest.com/services/ earthquake/html/eqengineering.html $\rangle$.

Moss, R. E. S., Cetin, K. O., and Seed, R. B. (2003a). "Seismic liquefaction triggering correlations within a Bayesian framework." Proc., 9th Int. Conf. Application of Statistic and Probability in Civil Engineering, Berkeley, Calif.

Moss, R. E. S., Seed, R. B., Kayen, R. E., Stewart, J. P., Youd, T. L., and Tokimatsu, K. (2003b). "Field case histories for CPT-based in situ liquefaction potential evaluation." Geoengineering Research Rep. $U C B / G E-2003 / 04$. 〈http://www.fugrowest.com/services/earthquake/ html/eqengineering.html .

Moss, R. E. S., Seed, R. B., and Olsen, R. S. (2006). "Normalizing the CPT for overburden stress." J. Geotech. Geoenviron. Eng., in press.

Olsen, R. S. (1984). "Liquefaction analysis using the cone penetrometer test." Proc., 8th World Conf. on Earthquake Engineering EERI, San Francisco, 247-254.

Olsen, R. S., and Koester, J. P. (1995). "Prediction of liquefaction resistance using the CPT." Proc., Int. Symp. on Cone Penetration Testing, CPT 95, Linkoping, Sweden, 251-256.

Olsen, R. S., and Mitchell, J. K. (1995). "CPT stress normalization and prediction of soil classification." Proc., Int. Symp. on Cone Penetration Testing, CPT 95, Linkoping, Sweden, 257-262.

Ooi, E. T. C. (1987). "Investigation of liquefaction in the Buller region." Master's thesis, Univ. of Canterbury, Christchurch, New Zealand.

Pacific Earthquake Engineering Research (PEER). (2000a). "Documenting incidents of ground failure resulting from the Aug. 17, 1999, Kocaeli, Turkey Earthquake."〈http://peer.Berkeley.edu〉.

Pacific Earthquake Engineering Research (PEER). (2000b). "Documentation of soil conditions at liquefaction sites from 1999 Chi-Chi, Taiwan Earthquake." 〈http://www.cee.ucla.edu/faculty/Taiwanwebpage/ Main.htm〉.

Perlea, V. G. (2000). "Liquefaction of cohesive soils." Soil dynamics and liquefaction 2000, ASCE Geotechnical Specialty Publication No. 107, ASCE, New York, 58-75.

Polito, C. (2001). "Plasticity based liquefaction criteria." Proc., 4th Int. Conf. Recent Advances in Geotechnical Earthquake Engineering and Soil Dynamics, Paper No. 1.33, Univ. Missouri-Rolla, Mo.

Reyna, F. A. M. (1991). "In situ tests for liquefaction potential evaluation: Application to California data including data from the 1989 Loma Prieta Earthquake.” Ph.D. dissertation, Purdue Univ., West Lafayette, Ind.

Robertson, P. K., and Campanella, R. G. (1985). "Liquefaction potential of sand using the CPT." J. Geotech. Eng., 111(3), 384-403.

Robertson, P. K., and Fear, C. E. (1995). "Liquefaction of sands and its evaluation." Proc., 1st Int. Conf. on Earthquake Geotechnical Engineering, K. Ishihara, ed., A. A. Balkema, Rotterdam, The Netherlands, 1253-1289.

Robertson, P. K., and Wride, C. E. (1998). "Evaluating cyclic liquefaction potential using the cone penetration test." Can. Geotech. J., 35(3), $442-459$.

Rollins, K. M., Hryciw, R. D., Shewbridge, S. E., McHood, M. D., and Homolka, M. (1994). "Ground response on Treasure Island." U.S. Geol. Surv. Prof. Pap., 1551.

Rongxiang, Z., and Zhaoji, S. (1995). "Liquefaction prediction based on $q_{c}$ and $D_{50} . "$ Earthquake Eng. Eng. Vibration, 15(1), 100-110.

Rutherford and Chekene. (1987). "Geotechnical hazard evaluation: Moss Landing Facility, Monterey Bay Aquarium Research Institute." Rep. Prepared for Monterey Bay Aquarium Research Institute, San Francisco.

Rutherford and Chekene. (1988). "Geotechnical investigation: Moss Landing Facility (technology building), Monterey Bay Aquarium Research Institute." Rep. Prepared for Monterey Bay Aquarium Research Institute, San Francisco.

Salgado, R., and Randolph, M. F. (2001). "Analysis of cavity expansion in sand." Int. J. Geomech., 1(2), 175-192.

Sancio, R. B., et al. (2002). "Correlation between ground failure and soil conditions in Adapazari, Turkey." Soil Dyn. Earthquake Eng., 22(9-12), 1093-1102

Schmertmann, J. H. (1978). "Guidelines for cone penetration performance and design." FHWA-TS-78-209, U.S. Dept. Transportation.

Seed, H. B. (1979). "Soil liquefaction and cyclic mobility evaluation for level ground during earthquakes." J. Geotech. Eng. Div., Am. Soc. Civ. Eng., 105(2), 201-255.

Seed, H. B., and De Alba, P. (1986). "Use of SPT and CPT tests for evaluating the liquefaction resistance of sands." Proc., In Situ '86, ASCE, New York, 281-302.

Seed, H. B., and Idriss, I. M. (1971). "Simplified procedure for evaluating soil liquefaction potential." J. Soil Mech. Found. Div., 97(9), 1249-1273.

Seed, H. B., and Idriss, I. M. (1982). Ground motion and soil liquefaction during earthquakes, Monograph, Earthquake Engineering Research Institute, Oakland, Calif.

Seed, H. B., Idriss, I. M., and Arango, I. (1983). "Evaluation of liquefaction potential using field performance data." J. Geotech. Eng., 109(3), 458-482.

Seed, R. B., et al. (2003). "Recent advances in soil liquefaction engineering: A unified and consistent framework." Rep. EERC 2003-06, Earthquake Engineering Research Center (http://eerc.berkeley.edu/ reports/>.

Shibata, T., Oka, F., and Ozawa, Y. (1996). "Characteristics of ground deformation due to liquefaction." Soils Found., 65-79.

Shibata, T., and Teparaska, W. (1988). "Evaluation of liquefaction potential of soils using cone penetration testing." Soils Found., 28(2), 49-60.

Stark, T. D., and Olson, S. M. (1995). "Liquefaction resistance using CPT and field case histories." J. Geotech. Eng., 121(12), 856-869.

Stewart, J. P. (2001). "Chapter 4: Soil liquefaction. Chi-Chi, Taiwan Earthquake of September 21, 1999 Reconnaissance Report." Earthquake Spectra, 17, Supplement A, 37-60.

Sugawara, N. (1989). "Empirical correlation of liquefaction potential 
using CPT." Proc., 12th Int. Conf. on Soil Mechanics and Foundation Engineering, Rio de Janeiro, Brazil, 335-338.

Suzuki, M., Tokimatsu, K., Moss, R. E. S., Seed, R. B., and Kayen, R. E. (2003). "CPT-based liquefaction field case histories from the 1995 Hyogoken-Nambu (Kobe) Earthquake, Japan.” Geotechnical Engineering Research Rep. UCB/GE-2003/03, Berkeley, Calif.

Suzuki, Y., Koyamada, K., Tokimatsu, K., Taya, Y., and Kubota, Y. (1995). "Empirical correlation of soil liquefaction based on cone penetration test." Proc., 1st Int. Conf. Geotechnical Earthquake Engineering, K. Ishihara, ed., A. A. Balkema, Rotterdam, The Netherlands, 369-374.

Toprak, S., Holzer, T. L., Bennett, M. J., and Tinsley, J. C. I. (1999). "CPT- and SPT-based probabilistic assessment of liquefaction." Proc., 7th U.S.-Japan Workshop on Earthquake Resistant Design of Lifeline Facilities and Countermeasures against Liquefaction, MCEER, Seattle, 69-86.

Vreugdenhil, R., Davis, R., and Berrill, J. R. (1994). "Interpretation of cone penetration results in multilayered soils." Int. J. Numer. Analyt. Meth. Geomech., 18, 585-599.

Wang, W. (1979). "Some findings in soil liquefaction." Research Rep., Water Conservancy and Hydroelectric Power Scientific Research Institute, Beijing.

Woodward-Clyde Consultants. (1990). "Phase I-Geotechnical study, Marine Biology Laboratory, California State University, Moss Land- ing, California." Rep. Prepared for California State Univ., San Jose, California, Oakland, Calif.

Yamamuro, J. A., and Lade, P. (1998). "Steady-state concepts and static liquefaction of silty sands." J. Geotech. Geoenviron. Eng., 124(9), 868-877.

Youd, T. L., and Garris, C. T. (1995). "Liquefaction-induced groundsurface disruption." J. Geotech. Eng., 121(11), 805-809.

Youd, T. L., and Gilstrap, S. D. (1999). "Liquefaction and deformation of silty and fine-grained soils." Proc., 2nd Int. Conf. Earthquake Geotechnical Engineering, Balkema, Rotterdam, The Netherlands, Vol. 3, 1013-1020.

Youd, T. L., and Wieczorek, G. F. (1984). "Liquefaction during the 1981 and previous earthquakes near Westmorland, CA." Open-File Rep. No. 84-680, U.S.G.S., Menlo Park, Calif.

Youd, T. L., et al. (2001). "Liquefaction resistance of soils: Summary report from the 1996 NCEER and 1998 NCEER/NSF workshops on evaluation of liquefaction resistance of soils." J. Geotech. Geoenviron. Eng., 127(10), 817-833.

Yu, H. S. (2000). Cavity expansion methods in geomechanics, Kluwer Academic, Dordrecht, The Netherlands.

Zhao, J. X., Dowrick, D. J., and McVerry, G. H. (1997). “Attenuation of peak ground accelerations in New Zealand earthquakes." New Zealand Nat. Soc. Earthquake Eng. Bull, 30(2), 133-158.

Zhou, S. G., and Zhang, S. M. (1979). "Liquefaction investigation in Tangshan District." Report to Ministry of Railway, China (in Chinese). 